\title{
Understanding the Uncertainty of Interatomic Potentials' Parameters and Formalism
}

\author{
A. P. Moore ${ }^{1,2}$, C. Deo ${ }^{1}$, M. I. Baskes ${ }^{3,4,5}$, Maria Okuniewski ${ }^{6,7}$, and D. L. McDowell ${ }^{8}$ \\ 1 Nuclear and Radiological Engineering Program, George W Woodruff School of Mechanical Engineering, Georgia Institute of Technology, 770 \\ State Street, Atlanta, GA 30332, USA \\ 2 Sandia National Laboratories, P.O. Box 5800, Albuquerque NM, 87185, USA \\ 3 Mississippi State University, 105 Lee Boulevard, MS 39762, USA \\ 4 University of California, San Diego, 9500 Gilman Drive, La Jolla, CA 92093, USA \\ 5 Los Alamos National Laboratory, PO Box 1663, Los Alamos, NM 87545, USA \\ 6 Purdue University, 610 Purdue Mall, West Lafayette, IN 47907, USA \\ 7 Idaho National Laboratory, PO Box 1625, Idaho Falls, ID 83415, USA \\ 8 Mechanical Engineering Program, George W Woodruff School of Mechanical Engineering, Georgia Institute of Technology, 770 State Street, \\ Atlanta, GA 30332, USA
}

\begin{abstract}
A sensitivity analysis of the modified embedded atom method (MEAM) potential for bodycentered-cubic uranium and zirconium was performed in order to examine and understand the uncertainty in the parameters and formalism of the interatomic potential. The sensitivity analysis was conducted using one-at-a-time (OAT) sampling of the parameters and how they affected the ground state, thermal, and alloy structural and thermodynamic properties. The performed analysis was able to uncover the properties that can be easily varied or adjusted like the lattice constant, and the properties that had little variance like the heat capacity. The observed analysis on the ground state properties was found to correspond well with previously published results, after which the thermal and alloy properties were examined. A new method of categorizing changes in the alloy properties was developed that allows for the discrimination of bonding behaviors, determining if the strength of the bonding between atoms changed or if the manner in which they were bonded together changed.
\end{abstract}

Keywords: Uncertainty, Sensitivity Analyses, Atomistic Modeling, bcc metals, MEAM potential

\section{Introduction}

Analytical Interatomic Potentials (AIPs) are mathematical formulae that provide a means to compute the potential energy of an ensemble of atoms with known spatial co-ordinates (1-4) and are widely used as the physical basis of molecular dynamics and Monte Carlo simulations in materials science.

While several standard potential functions have emerged for particular classes of systems (5), at present there is no definitive functional form that adequately describes all types of multiatom bonding. Instead, potentials are often developed for specific applications with functions and parameters determined on an ad hoc basis. In the past, practitioners (2) have often referred to the procedure of developing interatomic potentials as being as much as an art as a science. Recently, several quantitative procedures have been developed that aim to automate the process of developing interatomic potentials and that ensure fidelity of the analytics potential with respect to more accurate quantum mechanical calculations (6-10). Even so, there exists considerable and justified uncertainty with regard to the reliability of quantitative results produced by molecular 
dynamics simulations of materials based on analytic potentials. Such uncertainty is rarely explored, qualitatively or quantitatively.

Uncertainties in such simulations may be investigated by sensitivity analysis (SA), which investigates the connection between inputs and outputs of a (computational) model. The objective of SA is to identify how the variability in an output quantity of interest is connected to an input in the model. SA allows the practitioner to build a ranking of the input sources, which might dominate the response of the system. Note that strong large sensitivities derivatives do not necessarily translate in critical uncertainties because the input variability might be very small in a specific device of interest. Sensitivity analysis is thus usually considered to be a subset of uncertainty quantification methods (11-13).

In the case of AIPs, inputs for SA are parameters that have been chosen by the practitioner to represent the materials system of interest while outputs are specific material properties that are of interest. Usually, these properties are ground state quantities used in the fitting procedure that describe the material of interest such as the lattice parameter, cohesive energy, and vacancy formation energy at $0 \mathrm{~K}$. However, an intriguing feature of an interatomic potential is its transferability (14) - the ability to predict properties that have not been used in the fitting procedure. These may be thermodynamic and or mechanical properties of the material at ambient and high temperature where atomic motion introduces some variability in the computation of properties. Further the material system may be composed of multiple elements (e.g. alloys) that exhibit ordering or disordering tendencies. A comprehensive procedure to perform sensitivity analysis of interatomic potentials in such situations is lacking and is addressed in this work.

We choose, as an example on which to test our SA procedure, a recently developed interatomic potential of uranium zirconium alloys $(15,16)$. This potential is based on the Modified Embedded Atom Method (MEAM) (17), which is an extension of the Embedded Atom Method (EAM) (18). The basis of both EAM and MEAM formalisms is that the cohesive energy can be expressed in terms of embedding energies. In this view, each atom in the metal is embedded into the electron gas created by the other atoms. In addition, the MEAM formalism includes the ability to model atomic systems that exhibit directional bonding that is often found in metals and alloys that have complex non-cubic crystal structures.

Neither $\mathrm{U}$ or Zr, nor their alloys lend themselves easily to atomistic analysis. Uranium is an actinide that has three distinct stable solid phases. The transition between these phases can be attributed to the behavior of the de-localized $f$ orbital-electrons. The ground state uranium phase is the $\alpha$ (orthorhombic) phase. As temperature increases, uranium will first transition to the $\beta$ (tetragonal) and then to the $\gamma$ (body-centered-cubic) phases with transition temperatures 940.85 $\mathrm{K} \pm 1.3 \mathrm{~K}$ ( $\alpha$ to $\beta), 1047.95 \mathrm{~K} \pm 1.6 \mathrm{~K}(\beta$ to $\gamma)$, and $1405.95 \mathrm{~K} \pm 0.8 \mathrm{~K}$ ( $\gamma$ to liquid) (19). Zirconium is a transition metal with two distinct solid phases: the ground state $\alpha$ hexagonally closed packed (hcp) phase, and the $\beta$ high temperature body centered cubic (bcc) phase (above $1139 \mathrm{~K}$ ). The U-Zr alloy transitions from a $\delta$ (partially ordered C32 crystal structure) to $\gamma$ (bcc) phase around $65 \%-75 \% \mathrm{Zr}$ and $890 \mathrm{~K}$. Using atomistic simulations we have previously analyzed small configurations of $\mathrm{U}-\mathrm{Zr}$ alloys and looking for thermodynamic driving forces and unit mechanisms of ordering and phase separation (16).

In this work, the sensitivity of the MEAM potential on the ground state and thermal properties are thoroughly examined. The sensitivity analysis is conducted using the uranium, zirconium, and uranium-zirconium MEAM potentials. Sensitivity of ground state as well as thermal properties is conducted. Previous $0 \mathrm{~K}$ sensitivity analyses have been performed using 
MEAM potentials (20-22). In addition, there have also been attempts to quantify MEAM uncertainty using confidence intervals around specific values to obtain an uncertainty interval for various ground state properties $(23,24)$. To our knowledge, this is the first sensitivity analysis for elemental thermal properties and alloys, for which a new method of analyzing the effects that parameters have on the alloy properties has been developed. Such sensitivity analyses are helpful in potential development and the understanding of these interatomic potentials.

The atomistic simulations were conducted using molecular statics and molecular dynamic simulations on the MEAM U-Zr system. The molecular statics/dynamics (MS/MD) code DYNAMO (25) was used to perform all the atomistic simulations. The MD simulations performed in this research, unless otherwise specified, were conducted with a supercell consisting of 10x10x10 unit cells held in an isothermal-isobaric (NPT) ensemble. The thermal MD simulations were performed for 100 ps with a time step of $2 \mathrm{fs}$ with time averaged properties obtained over the last 35 ps. Alloy samples were constructed in random atomic configurations.

The second nearest neighbor 2NN MEAM potential theory is described in Appendix A, and is the formalism used during this sensitivity analysis. First, the elemental MEAM parameters are examined on how they change the ground state properties of the bcc phase and relative phase stability, and are compared to previous sensitivity analyses. In these sensitivity analyses, the MEAM parameters are varied one at a time, after which the effect that the change has on the properties is examined. This is followed by the elementary MEAM parameter thermal property sensitivity analyses, in which the heat capacity, lattice constant, thermal expansion and melting point are examined. In particular, some of the more complex analysis concerns how the interatomic potentials behave with thermal properties. Then the elementary and alloy MEAM parameters' effects on the alloy properties like Vegard's law for lattice constants and the enthalpy of mixing is examined. Since this is the first reported sensitivity analysis for thermal and alloy properties, a new method of quantifying how a change in a parameter affects the thermal and alloy response/properties was developed.

\section{Results and Discussion}

\section{Sensitivity Analysis Methodology}

A sensitivity analysis was performed on the degree to which the MEAM parameters affect the ground state and thermal properties of the elemental and alloy systems. In the past, there have been a few sensitivity analyses of the MEAM potential performed on ground state properties $(20,22,26)$, but this is the first reported sensitivity analysis of the MEAM potential performed on the thermal properties, which tend to be more complex and somewhat chaotic due to their nonindependent spatial parameters causing fluctuations. The thermal motion combined with the complexity of the angular partial electron densities and the screening parameters does not allow for a directly quantitative sensitivity analysis. Therefore, a semi-quantitative approach was used to describe the potential significance of how a change in the MEAM parameters could affect the ground state and thermal properties of the system. The sensitivity analysis performed uses one-at-a-time (OAT) sampling (13) where one parameter changes values between consecutive simulations, after which the results are analyzed. However, the input parameters are non-independent input factors creating seemingly random fluctuations or jumps over ranges of input parameters. In addition, the range of acceptable inputs for the parameters in this case is unknown, since changes in the parameters may lead to a destabilization of the phase of interest. Therefore, the maximum parameter change examined is set to be a percentage of the initial 
parameter value, and after each simulation the phase stability is examined ensuring the input change did not cause phase changes.

The OAT sampling is computationally expensive, therefore, the sensitivity analysis was conducted fully on the uranium potential, after which a more detailed analysis was conducted on the parameters determined to be significant with both the $\mathrm{U}$ and $\mathrm{Zr}$ potential.

\section{Effects of Elemental MEAM Parameters on Ground State Properties}

We begin by examining the ground state sensitivity analysis and comparing it to those previously published. The bcc phase elastic constants and bulk modulus are calculated, as well as the relative phase stability in terms of change in energy. Molecular statics was used to obtain the ground state material properties with an energy convergence criterion of $10^{-6}$. The elastic constants were calculated at $0 \mathrm{~K}$ via analysis of the changes in the internal energy due to small strains enforced on the simulation cell. The internal energy of the crystal system under strain was expanded in a Taylor series in powers of the strain tensor with respect to the initial energy of the unrestrained crystal. Then each elastic constant was computed from the application of a unique strain on the crystal and the resulting change in internal energy (27). This methodology is outlined by Soderlind (28).

It should be noted that the elemental MEAM parameter alat is often adjusted to directly fit the lattice constant of the phase of interest. Due to this direct fitting, a sensitivity analysis is not conducted on alat. The cohesive energy is often directly fit to a known value as well, however there are cases in which adjusting the cohesive energy away from the known value could improve other properties of interest and therefore a sensitivity analysis is conducted on the cohesive energy.

Table 1: bcc MEAM Sensitivity Table: Effect of U elemental parameters on elastic constants, bulk modulus, lattice constant, and relative phase energies.

\begin{tabular}{|c|c|c|c|c|c|c|c|c|c|c|c|c|c|}
\hline \multicolumn{14}{|c|}{ MEAM Parameters ( $15 \%$ change) } \\
\hline Properties & A & $\mathrm{B}^{0}$ & $B^{1}$ & $B^{2}$ & $B^{3}$ & $t^{1}$ & $t^{2}$ & $t^{3}$ & $C_{\text {min }}$ & $C_{\max }$ & $\delta$ & $\alpha$ & $E_{C}$ \\
\hline bcc $C_{11}$ and $C_{12}$ & + & - & / & - & / & / & - & / & - & - & + & + & + \\
\hline$(+>\Delta 2 \mathrm{GPa})$ & $\downarrow$ & $\uparrow$ & / & $\uparrow$ & / & / & $\uparrow$ & / & $\uparrow$ & $\uparrow$ & $\downarrow$ & $\uparrow$ & $\uparrow$ \\
\hline bcc $\mathrm{C}_{44}$ & + & + & / & + & / & / & + & / & - & - & - & + & + \\
\hline$(+>\Delta 2 \mathrm{GPa})$ & $\downarrow$ & $\downarrow$ & / & $\uparrow$ & / & / & $\uparrow$ & / & $\uparrow$ & $\uparrow$ & $\downarrow$ & $\uparrow$ & $\uparrow$ \\
\hline bcc Bulk Modulus & + & + & / & / & / & / & l & / & - & - & - & + & + \\
\hline$(+>\Delta 2 \mathrm{GPa})$ & $\downarrow$ & $\uparrow$ & / & / & / & / & l & / & $\downarrow$ & $\uparrow$ & $\downarrow$ & $\uparrow$ & $\uparrow$ \\
\hline bcc Lattice Constant & + & + & / & - & / & / & - & / & - & - & + & + & - \\
\hline$(+>0.001 \AA)$ & $\uparrow$ & $\uparrow$ & I & $\uparrow$ & I & I & $\uparrow$ & I & $\uparrow$ & $\downarrow$ & $\uparrow$ & $\downarrow$ & $\uparrow$ \\
\hline$\Delta \mathrm{Ebcc} \rightarrow \mathrm{fcc}$ & + & + & / & / & / & / & / & / & - & - & - & + & / \\
\hline (+> $\Delta 0.01 \mathrm{eV} /$ atom) & $\downarrow$ & $\uparrow$ & / & / & / & / & / & I & $\uparrow$ & $\downarrow$ & $\uparrow$ & $\downarrow$ & l \\
\hline$\Delta \mathrm{E}$ bcc $\rightarrow$ hcp & + & + & / & - & - & / & - & - & - & - & - & + & l \\
\hline (+> $0.01 \mathrm{eV} /$ atom) & $\downarrow$ & $\uparrow$ & / & $\uparrow$ & $\downarrow$ & / & $\uparrow$ & $\uparrow$ & $\uparrow$ & $\downarrow$ & $\uparrow$ & $\downarrow$ & / \\
\hline
\end{tabular}

In Table 1 above, the "+" sign means that the effect of a 15\% parameter change was significant, while a "-" sign means the change was less significant, and the "/" symbol means there was either no effect or almost no effect. The effects of the parameter changes on the bcc 
elastic constants, lattice constant, and bulk modulus $\left(\mathrm{C}_{11}, \mathrm{C}_{12}, \mathrm{C}_{44}\right.$, a, and $\left.\mathrm{B}\right)$ were deemed to be significant if the resulting change was greater than $2 \mathrm{GPa}$ for the elastic constants and bulk modulus, and 0.001 Angstroms for the lattice constant, and the change in energy between phases was deemed to be significant if they resulted in an energy change greater than $0.01 \mathrm{eV} /$ atom.

In addition to the significance of the parameter change on the ground state properties, the directionality of the relationship is also noted for the $15 \%$ parameter variations. In the table above, a " $\uparrow "$ designated a directional relationship where an increase in the MEAM parameter resulted in an increase in the thermal property; a " $\downarrow$ " designated an indirect relationship where an increase in the MEAM parameter resulted in a decrease in the thermal property; and a " $\uparrow$ " designated a mixed relationship.

The ground state sensitivity analysis matches up fairly well with those of Lee et al. (26) for the bcc elastic constants and Kim et al. (20) for the change in energy between the phases. However, this time a directionality was applied as well as the significance. The analysis performed on other crystal phase elastic constants shows different trends indicating the ground state properties of different phases are often dependent on different parameters $(20,22)$.

Lee et al. (26) performed a sensitivity analysis on the ground state properties for bcc elements. In this publication it was found that the $\mathrm{A}, \mathrm{B}^{0}$, and $\mathrm{C}_{\min }$ parameters all had a significant impact on the elastic constants and the relative phase energies, yet the significance was never quantified in terns if degree of change or its direction. Similarly, we found that the A, $\mathrm{B}^{0}$, and $\mathrm{C}_{\min }$, as well as others, had an impact on the properties and attribute some of the difference in less versus more significant to the cut-off used as well as the potential. While Kim et al. (20) performed her sensitivity analyses on the hcp ground state phase, she also included the change in the energy between phases in her analysis. In that publication the $\mathrm{A}, \mathrm{B}^{0}$, and $\mathrm{t}^{3}$ parameters were found to cause significant changes in the energy difference between the hcp and bcc phases. Once again we find that our results are consistent with those of Kim et al. (20), where the same parameters were found to cause a change in the relative energies between the bcc and hcp phases.

\section{Effects of Elemental MEAM Parameters on Thermal Properties}

The MD simulations performed in this research, unless otherwise specified, were conducted with a supercell consisting of 10x10x10 unit cells held in an NPT ensemble. The thermal MD simulations were performed for 100 ps with a time step of 2 fs with time averaged properties obtained over the last 35 ps. Alloy samples were constructed in random atomic configurations.

The heat capacity was calculated by the average change in the enthalpy over the change in temperature between $1000 \mathrm{~K}-1400 \mathrm{~K}$ for bcc uranium and $1600 \mathrm{~K}-2100 \mathrm{~K}$ for bcc zirconium. The thermal expansion reported is the average instantaneous thermal expansion coefficient between $1000 \mathrm{~K}-1400 \mathrm{~K}$ for bcc uranium and $1600 \mathrm{~K}-2100 \mathrm{~K}$ for bcc zirconium. However, the lattice constant was calculated by taking the average unit cell size at $1273 \mathrm{~K}$. Lastly, the melting temperature of the potential was determined within $25 \mathrm{~K}$ increments using the interface method described by Moore et al. (15).

The OAT sampling was performed for the table below by implementing a $15 \%$ change, both in the positive and negative direction, in each MEAM parameter on the uranium potential. Then the system was analyzed on the significance of how the change in the MEAM parameter affected the specific heat, the melting temperature, and the thermal expansion of the system. 
Table 2: bcc MEAM Sensitivity Table: Effect of U elemental parameters on elemental thermal properties.

\begin{tabular}{|c|c|c|c|c|c|c|c|c|c|c|c|c|c|}
\hline \multicolumn{14}{|c|}{ MEAM Parameters (15\% change) } \\
\hline Properties & A & $B^{0}$ & $B^{1}$ & $B^{2}$ & $B^{3}$ & $t^{1}$ & $t^{2}$ & $t^{3}$ & $C_{\min }$ & $C_{\max }$ & $\delta$ & $\alpha$ & $\mathrm{E}_{\mathrm{c}}$ \\
\hline $\begin{array}{c}\text { Heat Capacity } \\
\text { averaged between }\end{array}$ & + & $-/+$ & - & - & - & - & - & - & $-/+$ & - & - & $-/+$ & + \\
\hline $\begin{array}{c}1000 \mathrm{~K}-1400 \mathrm{~K} \\
(+\geq 1 \mathrm{~J} / \mathrm{mol}-\mathrm{K}) \\
(-<1 \mathrm{~J} / \mathrm{mol}-\mathrm{K})\end{array}$ & $\begin{array}{l}\uparrow \\
L\end{array}$ & $\uparrow$ & $\uparrow$ & $\begin{array}{l}\downarrow \\
L\end{array}$ & $\downarrow$ & $\uparrow$ & $\begin{array}{l}\downarrow \\
\mathrm{L}\end{array}$ & $\downarrow$ & $\uparrow$ & $\uparrow$ & $\begin{array}{l}\downarrow \\
\mathrm{L}\end{array}$ & $\uparrow$ & $\downarrow$ \\
\hline $\begin{array}{l}\text { Thermal Expansion } \\
\text { averaged between }\end{array}$ & + & + & + & - & - & - & + & - & + & + & + & + & + \\
\hline $\begin{array}{l}1000 \mathrm{~K}-1400 \mathrm{~K} \\
(+\geq 0.0004 \%) \\
(-<0.0004 \%)\end{array}$ & $\begin{array}{l}\uparrow \\
\mathrm{L}\end{array}$ & $\downarrow$ & $\uparrow$ & $\downarrow$ & $\uparrow$ & $\uparrow$ & $\begin{array}{l}\downarrow \\
\mathrm{L}\end{array}$ & $\uparrow$ & $\downarrow$ & $\begin{array}{l}\downarrow \\
L\end{array}$ & $\begin{array}{l}\uparrow \\
\mathrm{L}\end{array}$ & $\uparrow$ & $\downarrow$ \\
\hline $\begin{array}{l}\text { Lattice Constant } \\
\text { at } 1273 \mathrm{~K}\end{array}$ & + & + & - & - & - & - & - & - & + & + & + & + & + \\
\hline$(+\geq 0.1 \AA \AA)$ & $\uparrow$ & $\uparrow$ & $\uparrow$ & 个 & $\uparrow$ & $\downarrow$ & $\downarrow$ & $\uparrow$ & $\downarrow$ & & $\uparrow$ & $\downarrow$ & $\downarrow$ \\
\hline$(-<0.1 \AA)$ & L & & & $\mathrm{L}$ & & $\mathrm{L}$ & $\mathrm{L}$ & & $\mathrm{L}$ & $\mathrm{L}$ & $\mathrm{L}$ & $\mathrm{L}$ & $\mathrm{L}$ \\
\hline $\begin{array}{c}T_{\text {melt }} \\
(+\geq 100 \mathrm{~K})\end{array}$ & + & + & + & + & - & $-/+$ & $-/+$ & $-/+$ & + & + & $-/+$ & + & + \\
\hline$(-<100 \mathrm{~K})$ & $\downarrow$ & $\downarrow$ & $\uparrow$ & $\uparrow$ & $\uparrow$ & $\uparrow$ & $\uparrow$ & $\uparrow$ & $\uparrow$ & $\uparrow$ & $\downarrow$ & $\uparrow$ & $\uparrow$ \\
\hline
\end{tabular}

In Table 2 above, the "+" designates that the $15 \%$ change in the MEAM parameter caused a significant change in the thermal property; the "-" designates that the change was less significant; and the "-/+" designates that the change in the MEAM parameter had a significantly varying effect on the thermal property from less significant to greatly significant. A change in the elemental specific heat capacity was considered to be significant if greater than $1 \mathrm{~J} / \mathrm{mol}-\mathrm{K}$; a change in the melting temperature was considered significant if greater than $100 \mathrm{~K}$; a change in the thermal expansion was considered to be significant if greater than $0.0004 \%$; and a change in the lattice constant was considered significant if greater than 0.1 Angstroms.

In addition to the significance of the parameter change on the thermal properties, the directionality of the relationship is also noted for the $15 \%$ parameter variations. In Table 2 above, a " $\uparrow "$ designated a directional relationship where an increase in the MEAM parameter resulted in an increase in the thermal property; a " $\downarrow$ " designated an indirect relationship where an increase in the MEAM parameter resulted in a decrease in the thermal property; and a " $\uparrow "$ designated a mixed relationship. The " $\mathrm{L}$ " in the table indicates whether or not the $15 \%$ parameter variations resulted in a linear change in the property of interest. The trend was determined to be linear if $\mathrm{R}^{2}>0.90$ for the linear fit to three data points: the initial value, the $15 \%$ increased value, and the $15 \%$ decreased value. It should be noted that the melting point was determined between a range of values, making the determination of linearity of the melting point not feasible.

Since this table was constructed based on the bcc uranium and zirconium systems, it may give an expanded view of the sensitivity analysis of other bcc systems. However, due to the 
symmetry and complexities of the MEAM potential, it may not be applicable to other crystal structures.

The data in Table 2 show that while there are a few parameters that affect the elemental specific heat, they tend to be fitted based on the ground state properties and greatly affect both the thermal and ground state properties of the system. Therefore, the fitting of the elemental specific heat is not performed with the MEAM potentials, but normally falls into place after the other parameter fitting has been conducted. It should be noted that the cohesive energy parameter $E_{C}$ had the greatest impact on the specific heat capacity. Some of the $15 \%$ parameter changes in $\mathrm{B}^{0}$ and $\alpha$ caused obvious destabilizations in the bec phase and could not be considered.

The elemental melting temperature is affected by a great number of MEAM parameters, including cohesive energy, bulk modulus, the embedding function parameter A, the screening parameters, and the partial electron density parameters. However, the partial electron density parameters tended to have a wildly varying significance on the melting temperature.

The thermal expansion of the system was affected by the base parameters fitted from the ground state properties, as well as from some of the partial electron density parameters and the reference state parameter $\delta$, which is often used to fit the thermal expansion values.

To give a better view of how a few of the parameters influence the thermal properties, the parameter and its effect on the specific thermal property were plotted in the following figures. Each parameter was varied by $\pm 5 \%, \pm 15 \%$, and $\pm 25 \%$ of its initial value, giving seven data points. However, some of the parameter changes caused phase destabilization and therefore were not reported. Occasionally additional data points were added to give a better view of how the properties changed with the parameters.

Parameter sensitivity analysis can be categorized into parameters that significantly change local environment interactions like B's and $\mathrm{C}_{\min }$ and $\mathrm{C}_{\max }$, in contrast to the parameters that just scale current interactions, e.g., A. The distance parameters often cause more varied results due to the fact that changing a parameter affects how much the surrounding atoms contribute to the potential, as described below.

Bcc metals modeled with the MEAM potential tend to have a $\mathrm{t}^{3}$ parameter that is either very small or more likely negative, a $\mathrm{t}^{2}$ parameter that is positive, and a $\mathrm{t}^{1}$ parameter that is positive. These partial electron weighting parameters are important for the stability and structure of the MEAM potential. It has been observed that the $\mathrm{t}^{3}$ parameter can particularly destabilize the bcc phase if increased too much. This destabilization effect can be seen in the lowering of the melting temperature with the increasing $\mathrm{t}^{3}$ value. Similarly, a larger $\mathrm{B}^{3}$ value causes the partial electron density to decay more quickly and therefore leads to an increase in melting temperature and at the same time a decrease in the thermal expansion and heat capacity values. This opposite behavior is a common trend for the thermal properties. Elemental parameters that affect the thermal expansion and heat capacity normally oppositely affect the melting temperature. 


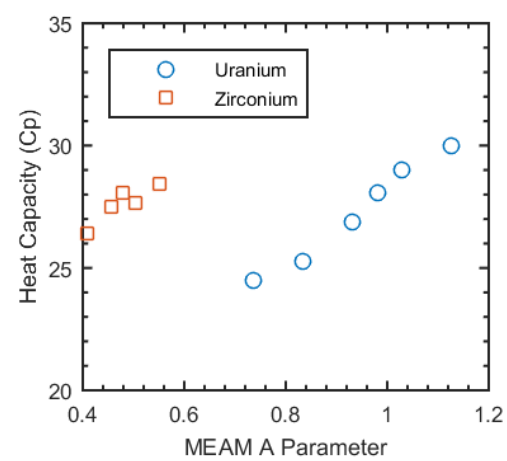

(a)

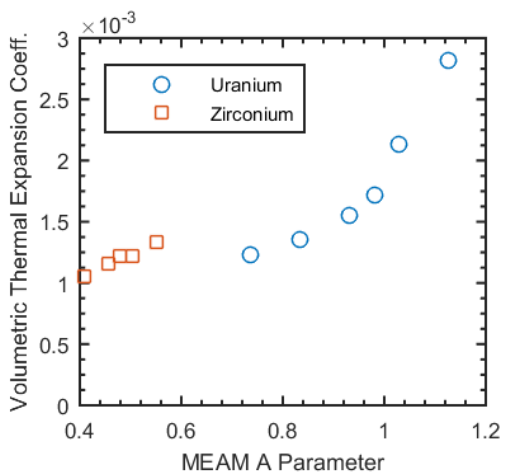

(b)

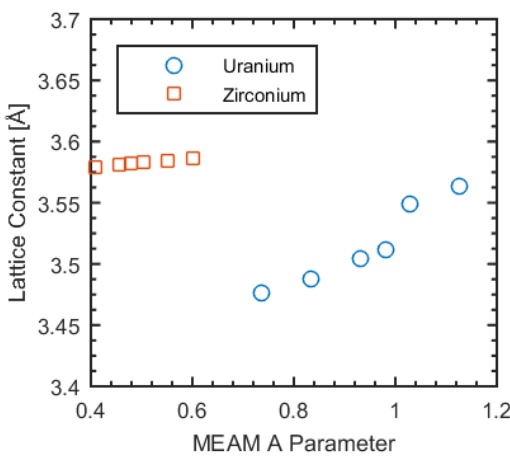

(c)

Figure 1: Sensitivity analysis of U's and Zr's MEAM parameter A on (a) $\mathrm{C}_{\mathrm{P}}$ between $1000 \mathrm{~K}$ $1400 \mathrm{~K}$, (b) volumetric thermal expansion between $1000 \mathrm{~K}-1400 \mathrm{~K}$, and (c) lattice constant at $1273 \mathrm{~K}$.

Figure 1 is an example of a sensitivity analysis of elemental parameter A, and its effect on some thermal properties. The parameter A is a constant multiplier to the embedding function, making it an important parameter to fit, but also one of the more simplistic one when it comes to the thermal properties, as it is not directly multiplied by either the radial pair interaction function or the radial screening function, causing the parameter have less of a radial distance dependence than many of the other parameters. The parameter and its effect on the heat capacity, thermal expansion and lattice constant can be seen in the graphs. It can be seen that an increase in the parameter A causes each of these properties to increase, often according to a mostly linear relationship.

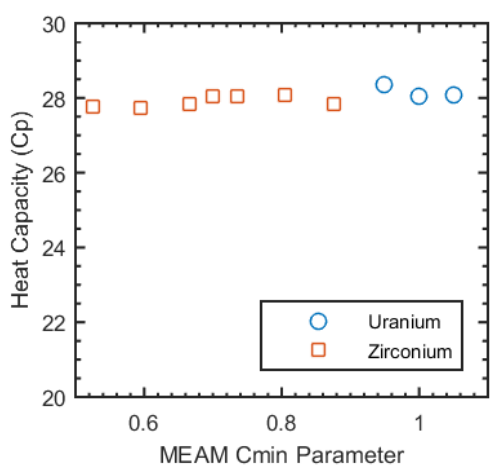

(a)

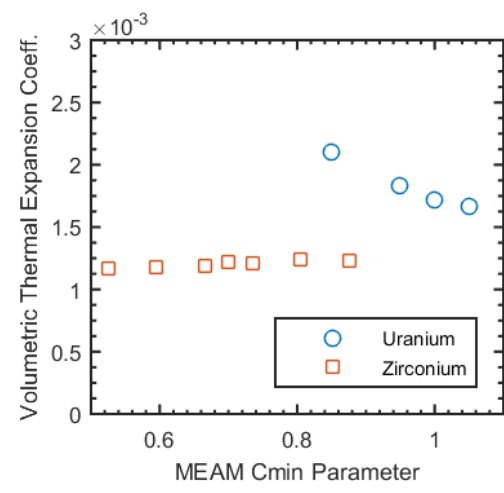

(b)

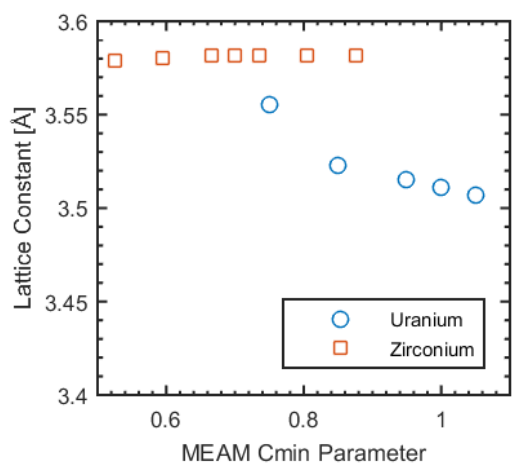

(c)

Figure 2: Sensitivity analysis of U's and Zr's MEAM parameter $\mathrm{C}_{\min }$ on (a) $\mathrm{C}_{\mathrm{P}}$ between $1000 \mathrm{~K}$ $1400 \mathrm{~K}$, (b) volumetric thermal expansion between $1000 \mathrm{~K}-1400 \mathrm{~K}$, and (c) lattice constant at $1273 \mathrm{~K}$.

Figure 2 is an example of a sensitivity analysis of elemental parameter $C_{\min }$, and its effect on some thermal properties. The parameter $\mathrm{C}_{\min }$ is part of the bounds of the screening function, which is multiplied to the pair potential, making it a very important parameter, but it also has a very complex relationship with thermal properties. Since $\mathrm{C}_{\min }$ is directly related to screening bounds, it directly affects how much each of the surrounding atoms contribute to the potential, making the effect on thermal properties dependent on the local environment. This is seen in the 
sensitivity analysis of the uranium and zirconium potentials, in that their trends do not agree with each other.

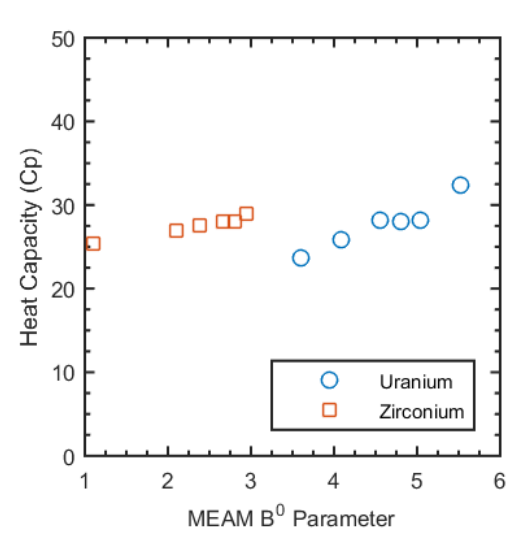

(a)

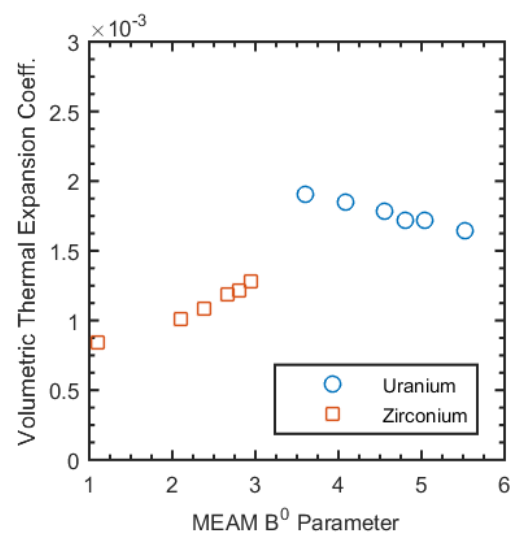

(b)

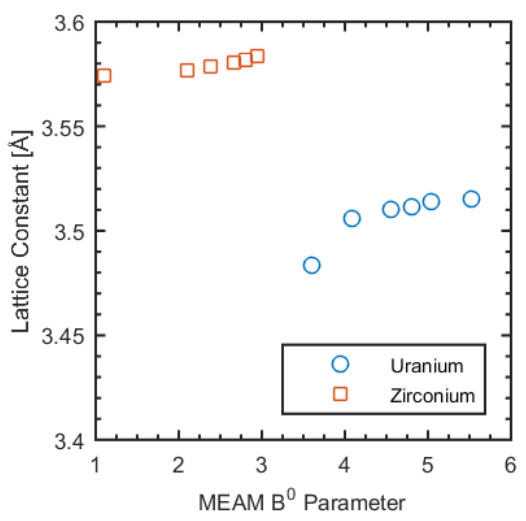

(c)

Figure 3: Sensitivity analysis of U's and Zr's MEAM parameter $B^{0}$ on (a) $C_{P}$ between $1000 \mathrm{~K}$ $1400 \mathrm{~K}$, (b) volumetric thermal expansion between $1000 \mathrm{~K}-1400 \mathrm{~K}$, and (c) lattice constant at $1273 \mathrm{~K}$.

Figure 3 is an example of a sensitivity analysis of elemental parameter $\mathrm{B}^{0}$, and its effect on some thermal properties. The parameter $\mathrm{B}^{0}$ is the distance decay function of the spherical partial electron density. Once again this parameter has a direct relationship with how much the surrounding atoms contribute to the potential, making the thermal property analysis more complex. In addition, it is often used to fit the relative phase energies. This analysis showed that the $\mathrm{B}^{0}$ parameter has a great effect on the bcc phase stability, since many of the parameter changes resulted in destabilizing the phase.

\section{Effects of Elemental MEAM Parameters on Alloy Thermal Properties}

Similar to the elemental thermal properties, a sensitivity analysis of the alloy's thermal properties was also performed. Once again a $15 \%$ change, both in the positive and negative direction, in each MEAM parameter was varied one at a time for the uranium potential. The system was analyzed regarding the significance of how the change in the MEAM parameter affected the alloy lattice constant versus zirconium mole fraction curve (or Vegard's law) and the enthalpy of mixing versus zirconium mole fraction curve. The enthalpy of mixing was obtained by using the basic definition of enthalpy and ensemble averages for volume (V), pressure (P), and internal energy (U). The pure $\mathrm{U}$ and $\mathrm{Zr}$ ensemble time-averaged properties at a given temperature were taken and set to the component properties (e.g. $\mathrm{P}_{\mathrm{i}}, \mathrm{V}_{\mathrm{i}}$, and $\mathrm{U}_{\mathrm{i}}$ ). The alloy's ensemble time-averaged properties for a given composition $(\mathrm{X})$ and temperature were taken and set to the "mixed" properties (e.g. $\mathrm{P}_{\text {mix }}, \mathrm{V}_{\text {mix }}$ and $\mathrm{U}_{\text {mix }}$ ). This method allows for a representative model of the MEAM potential on the enthalpy of mixing, given by

$\Delta H_{\text {mix }}=U_{\text {mix }}-\sum_{i} X_{i} U_{i}+P_{\text {mix }} V_{\text {mix }}+\sum_{i} X_{i} P_{i} V_{i}$

where for the MD simulations held in a NPT ensemble, the pressures $\mathrm{P}_{\text {mix }}$ and $\mathrm{P}_{\mathrm{i}}$ are approximately zero, while the lattice constants were obtained using the average unit cell box 
size. Previously created MEAM alloy potentials are often fitted to pure elastic constants using knowledge of the system, but use guess and check methods with thermal properties like lattice constants and enthalpy of mixing $(20,29-33)$. In order to solve this issue, a systematic sensitivity analysis of alloy's structural and thermodynamic properties was conducted on the U$\mathrm{Zr}$ system by examining the changes in $\mathrm{U}-20,40,60,80$ at.\% Zr.

Table 3: bcc MEAM Sensitivity Table: Effect of U elemental parameters on alloy thermal properties.

\begin{tabular}{|c|c|c|c|c|c|c|c|c|c|c|c|c|c|}
\hline \multicolumn{14}{|c|}{ MEAM Parameters ( $15 \%$ change) } \\
\hline Properties & A & $B^{0}$ & $\mathrm{~B}^{1}$ & $B^{2}$ & $B^{3}$ & $t^{1}$ & $t^{2}$ & $t^{3}$ & $C_{\text {min }}$ & $\mathrm{C}_{\max }$ & $\delta$ & $\alpha$ & $E_{C}$ \\
\hline $\begin{array}{c}\text { Vegard's } \\
(+P \text { if } a \geq 0.35)\end{array}$ & $+P$ & $+P$ & $-P$ & $-P$ & & $-P$ & $+P$ & & $-P$ & $-P$ & $-P$ & $+P$ & $+P$ \\
\hline (-P if .35>a $\geq .1)$ & $+S$ & $+S$ & $-S$ & $-S$ & $-S$ & $-S$ & $+S$ & $+S$ & $-S$ & $-S$ & $-S$ & $-S$ & $+S$ \\
\hline $\begin{array}{c}\left(+S \text { if } \Sigma\left|r_{i}\right| \geq .5\right) \\
\left(-S \text { if } .5>\Sigma\left|r_{i}\right| \geq .1\right)\end{array}$ & $\uparrow$ & $\uparrow$ & $\uparrow$ & $\uparrow$ & $\downarrow$ & $\uparrow$ & $\uparrow$ & $\uparrow$ & $\uparrow$ & $\uparrow$ & $\downarrow$ & $\uparrow$ & $\downarrow$ \\
\hline $\begin{array}{l}\text { Enthalpy of Mixing } \\
\qquad(+P \text { if } a \geq 0.04)\end{array}$ & $+P$ & $+P$ & / & $-P$ & I & $-P$ & $+P$ & & $-P$ & & $-P$ & $+P$ & $+P$ \\
\hline (-P if .04>a $\geq .01)$ & $+S$ & $+S$ & & $-S$ & & $-S$ & $+S$ & $+S$ & $-S$ & $-S$ & & $+S$ & $-S$ \\
\hline $\begin{array}{c}\left(+S \text { if } \Sigma\left|r_{i}\right| \geq .009\right) \\
\left(-S \text { if } .009>\Sigma\left|r_{i}\right| \geq .002\right)\end{array}$ & $\uparrow$ & $\downarrow$ & $\uparrow$ & $\downarrow$ & $\uparrow$ & $\uparrow$ & $\uparrow$ & $\uparrow$ & $\uparrow$ & $\uparrow$ & $\uparrow$ & $\downarrow$ & $\downarrow$ \\
\hline
\end{tabular}

Results of the sensitivity analysis performed on the alloy's lattice parameter versus concentration (or Vegard's law) and the enthalpy of mixing for the random solid solution are provided in Table 3. Once again the "-" and the "+" symbols are used to designate that the change in the MEAM parameter causes either a less significant or more significant change to the property in question. However, this time a "P", for parabola shifted, or an "S", for shape, was added to designate how the property over concentration curve varied once the parameter had been changed. The "P", or parabola shifted, meant that the property curve could be shifted from its initial position (before the parameter change) by adding or subtracting a symmetric parabola across the mole fraction to it final position (after the parameter change). The "S", or shape shifted curve, meant that the addition or subtraction of a symmetric parabola could not entirely encompass the change in the property over concentration curve. In other words, the " $\mathrm{S}$ " means that the shape of the property curve had changed.

The significance of a parameter change on the enthalpy of mixing was determined using Equation (2), where the change between the initial and final curves is deconstructed into a symmetric parabola and a shape function. First the change between the initial and final curves was deconstructed by determining the constant "a" that minimized the residuals, after which the resulting residuals determined comprise the shape function.

$\Delta H_{\text {mix,final }}\left(X_{Z r}\right)=\Delta H_{\text {mix,initial }}\left(X_{Z r}\right)+a *\left(1-X_{Z r}\right) * X_{Z r}+S\left(X_{Z r}\right)$

In Equation (2), "a" is a constant multiplier to the addition of the symmetric parabola, and $\mathrm{S}\left(\mathrm{X}_{\mathrm{Zr}}\right)$ is the additional change in shape function. The degree of significance of the change was determined by the constant multiplier "a" and the shape function, $\mathrm{S}\left(\mathrm{X}_{\mathrm{Zr}}\right)$. The value of the constant " $a$ " will determine if the bonding strength change is significant. After the parabola is 
fit, the resulting difference between the initial and final result is the shape function "S". The sum of the residuals comprising shape function " $S$ " determine the significance of how the atoms are bonded together. The change for the enthalpy of mixing was seen as significant if the "a" constant for the parabola was $>0.04$ or the sum of the residuals was greater than 0.009 .

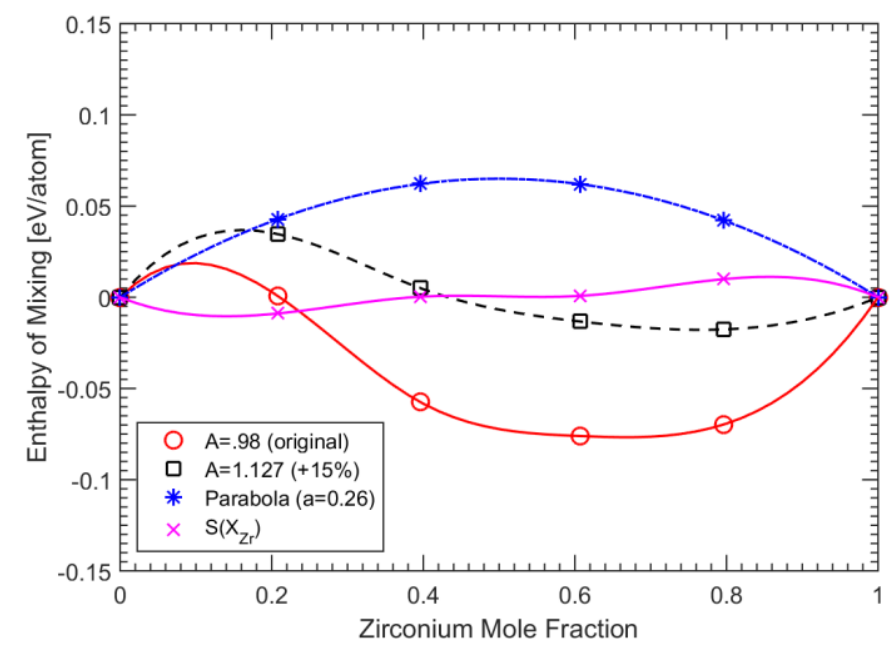

Figure 4: Visualization of the change in the enthalpy of mixing curve when U's A parameter is increased by $15 \%$ from 0.98 to 1.127 , and the determination that the addition of a symmetric parabola with $\mathrm{a}=0.26$ and a shape function $\mathrm{S}\left(\mathrm{X}_{\mathrm{Zr}}\right)$ can mimic the effect seen in the change of the enthalpy of mixing.

Figure 4 shows an example of the change in elemental parameter A for $U$ on the alloy enthalpy of mixing curve, and how it can be deconstructed into a symmetric parabola with the constant "a" equal to 0.26 and a shape function of $S\left(X_{Z} r\right)$. In this example, "a" being greater than 0.04 results in a $+\mathrm{P}$ relationship, and the sum of the residuals for the shape function being greater than 0.009 also results in a $+\mathrm{S}$ relationship.

However, for Vegard's law we had to separate the elemental thermal expansion effects from the lattice constant over concentration curve. Therefore, the lattice constant over concentration curve was first normalized between the starting point of 0 and the ending point of 1. This normalization allows for the analysis to be performed in a similar manner as the enthalpy of mixing, i.e.,

$\omega_{b c c, \text { inital }}\left(X_{Z r}\right)=\left(\frac{a_{b c c, \text { initial }}\left(X_{Z r}\right)-a_{b c c, \text { initial }}\left(X_{Z r}=0\right)}{a_{b c c, \text { initial }}\left(X_{Z r}=1\right)-a_{b c c, \text { initial }}\left(X_{Z r}=0\right)}\right)$
$\omega_{b c c, \text { final }}\left(X_{Z r}\right)=\left(\frac{a_{b c c, \text { final }}\left(X_{Z r}\right)-a_{b c c, \text { final }}\left(X_{Z r}=0\right)}{a_{b c c, \text { final }}\left(X_{Z r}=1\right)-a_{b c c, \text { final }}\left(X_{Z r}=0\right)}\right)$
$\omega_{b c c, \text { final }}\left(X_{Z r}\right)=\omega_{b c c, \text { initial }}\left(X_{Z r}\right)+a *\left(1-X_{Z r}\right) * X_{Z r}+S\left(X_{Z r}\right)$.

In Equation (3), $\omega$ was introduced as the temporary variable for the normalized lattice constant. The change for Vegard's law was seen as significant if the "a" constant for the parabola was greater than 0.35 or the sum of the residuals was greater than 0.04 .

Once again a more detailed analysis was conducted on the MEAM parameters that were determined to be significant, and their effects on the alloy properties. The parameters were varied by $\pm 5 \%, \pm 15 \%$, and $\pm 25 \%$ of its initial value starting with seven data points, minus the 
parameter changes that destabilized the phase. However, some of the parameter changes caused phase destabilization and therefore were not reported. All the reported properties were calculated at or around a $1100 \mathrm{~K}$ midpoint.

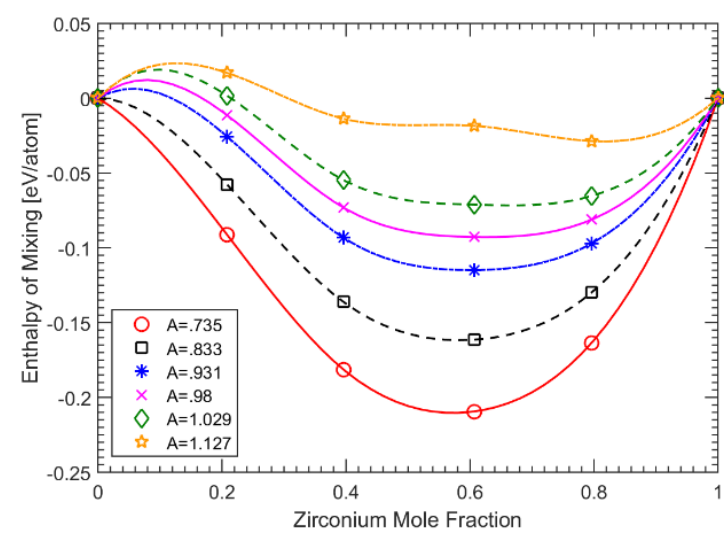

(a)

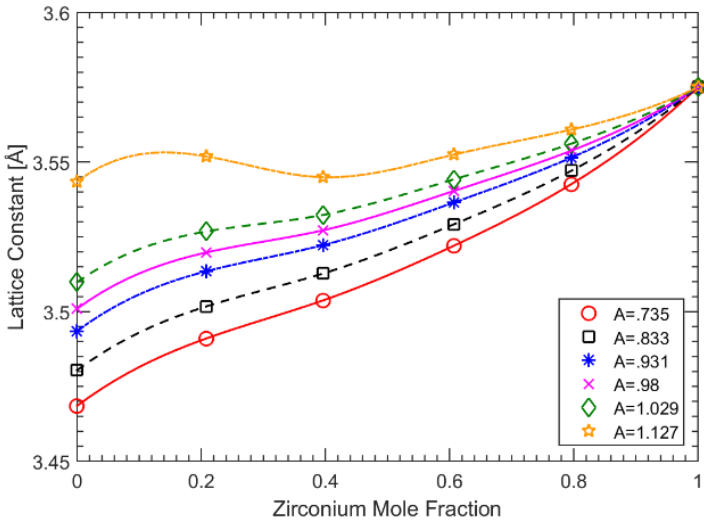

(b)

Figure 5: Sensitivity analysis of U's MEAM parameter "A" on (a) enthalpy of mixing and (b) Vegard's law.

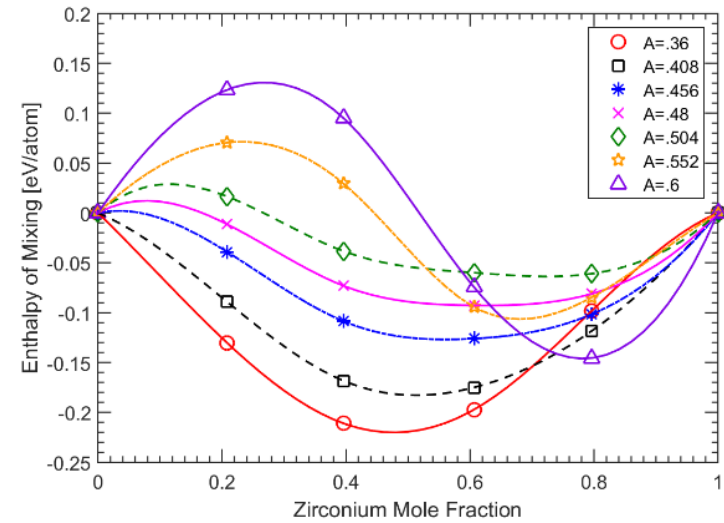

(a)

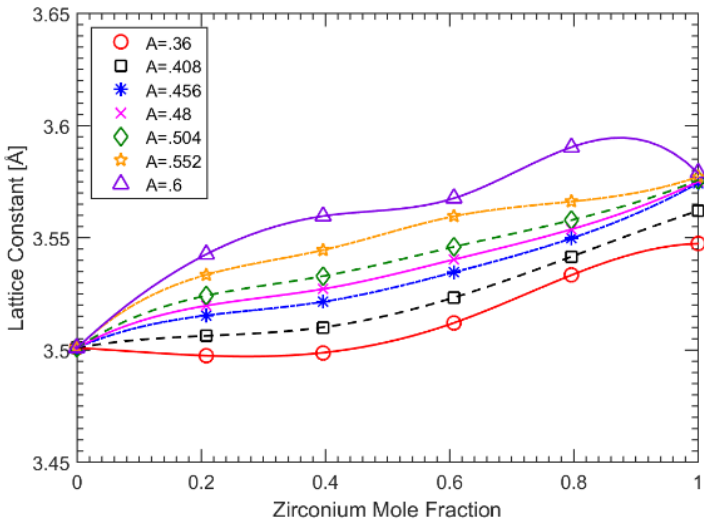

(b)

Figure 6: Sensitivity analysis of Zr's MEAM parameter "A" on (a) enthalpy of mixing and (b) Vegard's law.

Figure 5 and Figure 6 show how the elemental parameter "A" affects the alloy's thermal properties. It can be seen that the change in the parameter affects the change in both the enthalpy of mixing and the Vegard's law via a symmetric parabola, as well as changes the shape of the curve, indicating $\mathrm{a}+\mathrm{P}$ and $+\mathrm{S}$ relationship. In addition, generally an increase in the parameter caused the curve to shift into the more positive values, indicating an overall direct $(\uparrow)$ relationship. 


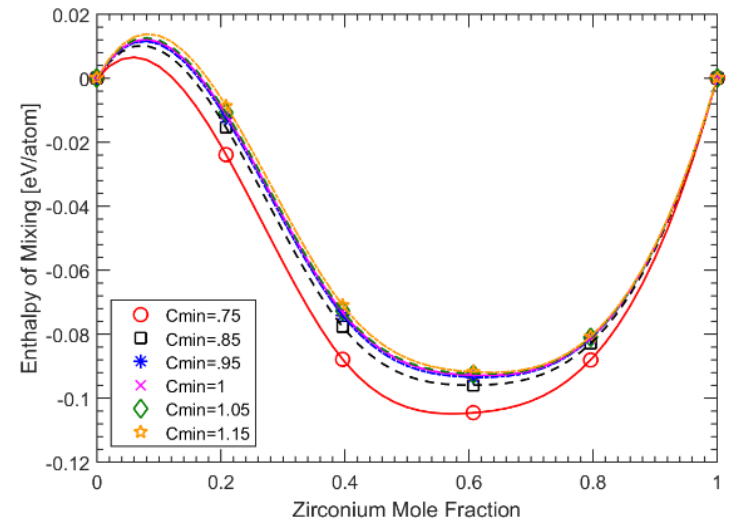

(a)

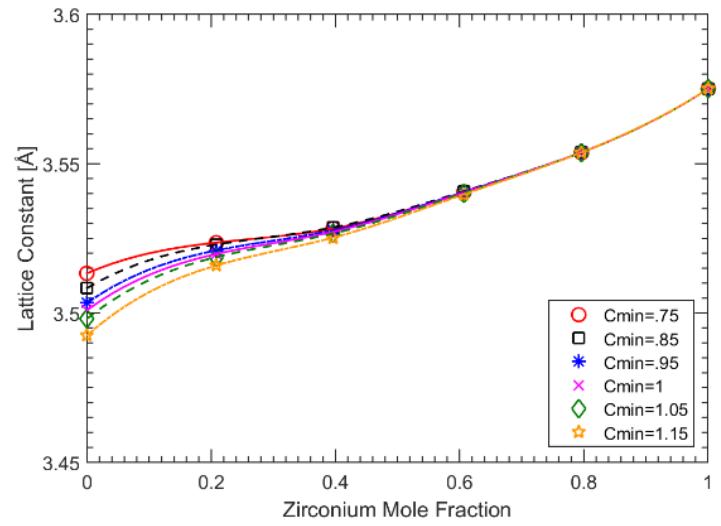

(b)

Figure 7: Sensitivity analysis of U's MEAM parameter $C_{\min }$ on (a) enthalpy of mixing and (b) Vegard's law.

Figure 7 shows how the elemental parameter $\mathrm{C}_{\min }$ of uranium affects the alloy's thermal properties. The zirconium $\mathrm{C}_{\min }$ parameter had even less of an effect on the properties and therefore was not plotted. It can be seen that the change in the parameter minimally affects the enthalpy of mixing and the Vegard's law. However, an increase in the parameter caused the enthalpy of mixing to slightly shift upward and the Vegard's law to shift downward, indicating a direct relationship for the enthalpy of mixing and a direct relationship for Vegard's law, once the thermal expansion effects were normalized.

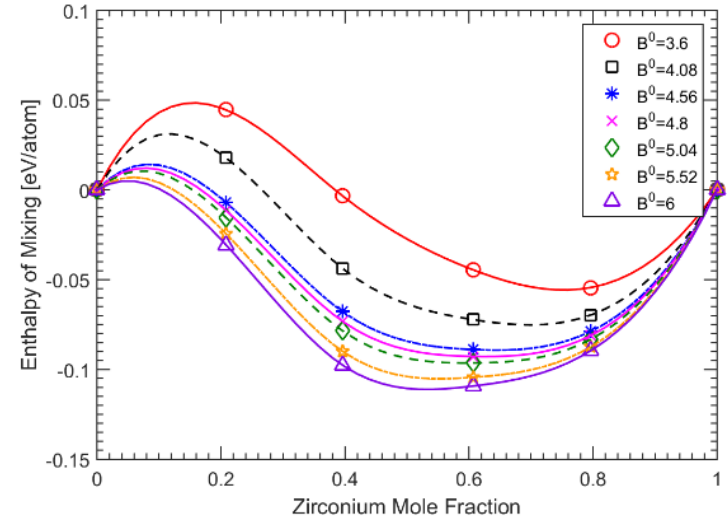

(a)

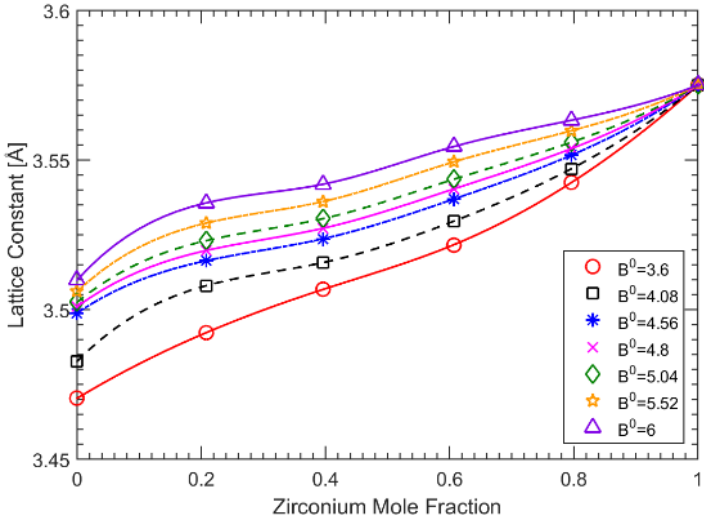

(b)

Figure 8: Sensitivity analysis of U's MEAM parameter $\mathrm{B}^{0}$ on (a) enthalpy of mixing and (b) Vegard's law. 


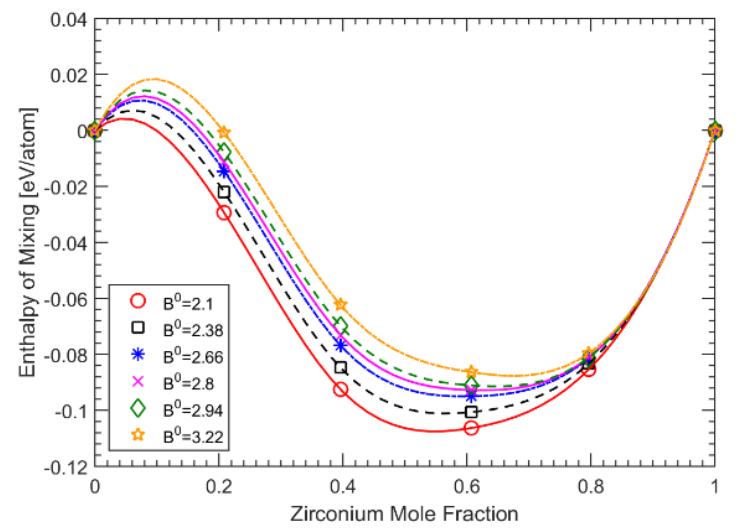

(a)

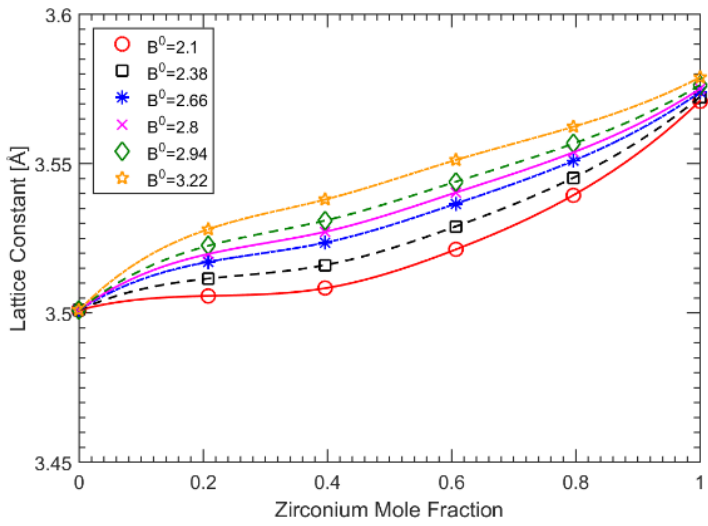

(b)

Figure 9: Sensitivity analysis of Zr's MEAM parameter $\mathrm{B}^{0}$ on (a) enthalpy of mixing and (b) Vegard's law.

Figure 8 and Figure 9 show how the elemental parameter $\mathrm{B}^{0}$ affects the alloy's thermal properties. It can be seen that the change in the parameter affects the change in both the enthalpy of mixing and the Vegard's law via a symmetric parabola, as well as changes the shape of the curve, indicating $\mathrm{a}+\mathrm{P}$ and $+\mathrm{S}$ relationship. In addition, generally an increase in the parameter caused the curve to shift into the more positive values, indicating an overall direct relationship.

\section{Effects of Alloy MEAM Parameters on Alloy Thermal Properties}

The effect of alloy MEAM parameters on the alloy's properties was also examined, again using a $15 \%$ change, both in the positive and negative direction, in each MEAM parameter. The system was analyzed regarding the significance of how the change in the MEAM parameter affected the alloy's lattice constant versus zirconium mole fraction curve (or Vegard's law) and the enthalpy of mixing versus zirconium mole fraction curve.

It can be seen that the alloy screening parameters $\left(\mathrm{C}_{\min }\right.$ and $\left.\mathrm{C}_{\max }\right)$ for the six local configurations of $\mathrm{U}$ and $\mathrm{Zr}$ atoms are not presented in Table 4. The paper from Moore et al. (15) briefly mentioned the influence of these alloy screening parameters on the enthalpy of mixing versus the partial electron density parameters. It was determined that the elemental partial electron density parameters played a larger role in the enthalpy of mixing than the alloy screening parameters. However, we do note that significant changes to the alloy screening parameters have the potential to destabilize the alloy bcc structure, and could possibly influence the preferential alloy ordering or plasticity pathways for alloys. Therefore, due to their less significant effect on the examined alloy thermal properties, their complexity, and their computational efficiency, the sensitivity analysis of these parameters was not presented. 
Table 4: bcc MEAM Sensitivity Table: Effect of alloy parameters on alloy thermal properties.

\begin{tabular}{|c|c|c|c|c|}
\hline \multicolumn{5}{|c|}{ MEAM Parameters (15\% change) } \\
\hline Properties & Alloy $\delta$ & Alloy $\alpha$ & Alloy $\Delta$ & $\rho^{0}$ \\
\hline $\begin{array}{c}\text { Vegard's } \\
(+P \text { if } a \geq 0.35) \\
(-P \text { if } .35>a \geq .1) \\
\left(+S \text { if } \Sigma\left|r_{i}\right| \geq .5\right) \\
\left(-S \text { if } .5>\Sigma\left|r_{i}\right| \geq .1\right)\end{array}$ & $+P$ & $+P$ & $+P$ & $+P$ \\
\hline $\begin{array}{c}\text { Enthalpy of Mixing } \\
(+P \text { if } a \geq 0.04) \\
(-P \text { if } .04>a \geq .01) \\
\left(+S \text { if } \Sigma\left|r_{i}\right| \geq .009\right)\end{array}$ & + & $\downarrow$ & $\uparrow$ & $\uparrow$ \\
$\left(-S\right.$ if $\left..009>\Sigma\left|r_{i}\right| \geq .002\right)$ & $\downarrow$ & $+P$ & $+P$ & $+P$ \\
\hline
\end{tabular}

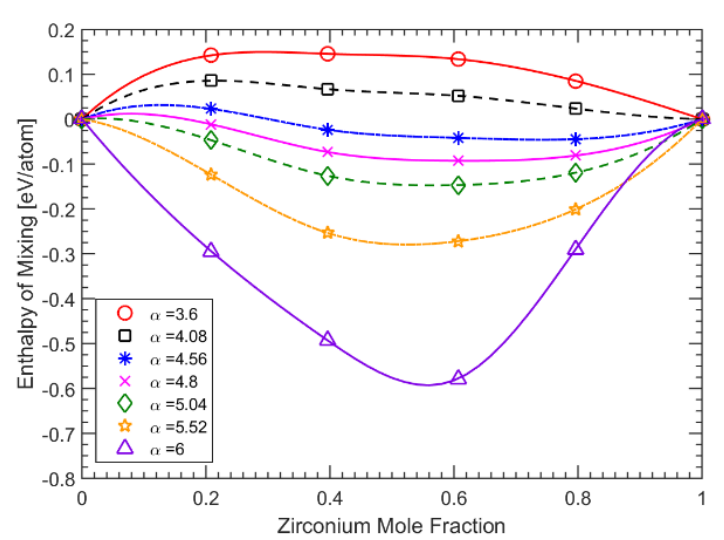

(a)

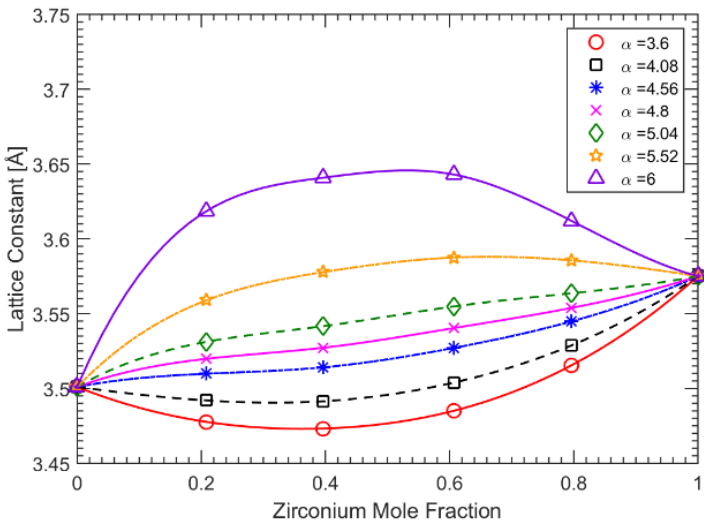

(b)

Figure 10: Sensitivity analysis of U-Zr alloy MEAM parameter $\alpha$ on (a) enthalpy of mixing and (b) Vegard's law.

Figure 10 shows how the alloy parameter $\alpha$ affects the alloy's thermal properties. It can be seen that the change in the parameter affects the change in both the enthalpy of mixing and the Vegard's law mostly via a symmetric parabola, and only slightly changes the shape of the curve, indicating $\mathrm{a}+\mathrm{P}$ and $-\mathrm{S}$ relationship. This parameter shows great potential for calculated MEAM alloy property adjustment using a symmetric parabola only. 


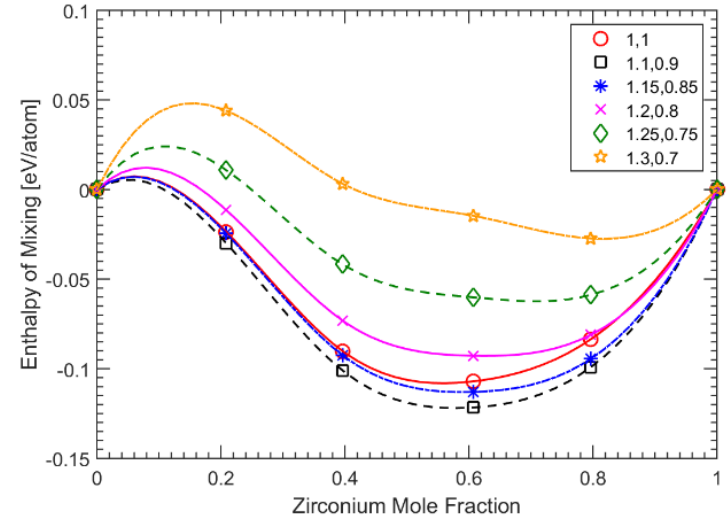

(a)

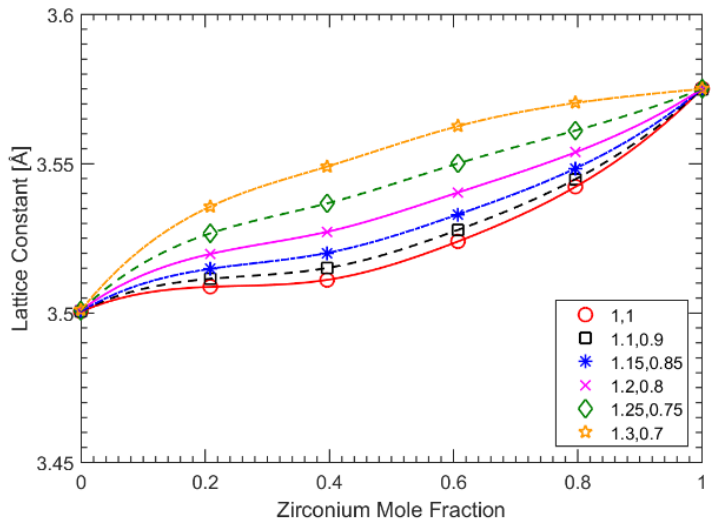

(b)

Figure 11: Sensitivity analysis of U-Zr alloy MEAM parameters $\rho_{U}, \rho_{Z r}$ on (a) enthalpy of mixing and (b) Vegard's law.

Figure 11 shows how the parameters $\rho_{\mathrm{U}}, \rho_{\mathrm{Zr}}$ affect the alloy's thermal properties. These parameters are often used to fit the alloy properties. It can be seen that the change in the parameter affects the change in both the enthalpy of mixing and the Vegard's law mostly via a symmetric parabola, and only slightly changes the shape of the curve, indicating a $+\mathrm{P}$ and $-\mathrm{S}$ relationship.

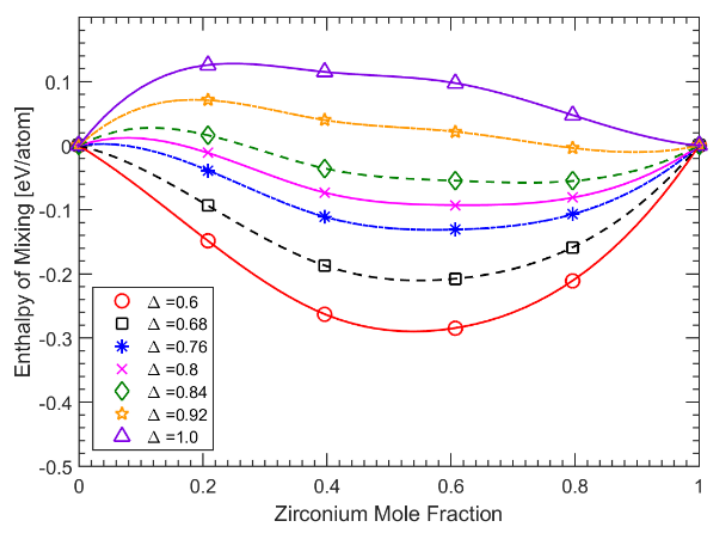

(a)

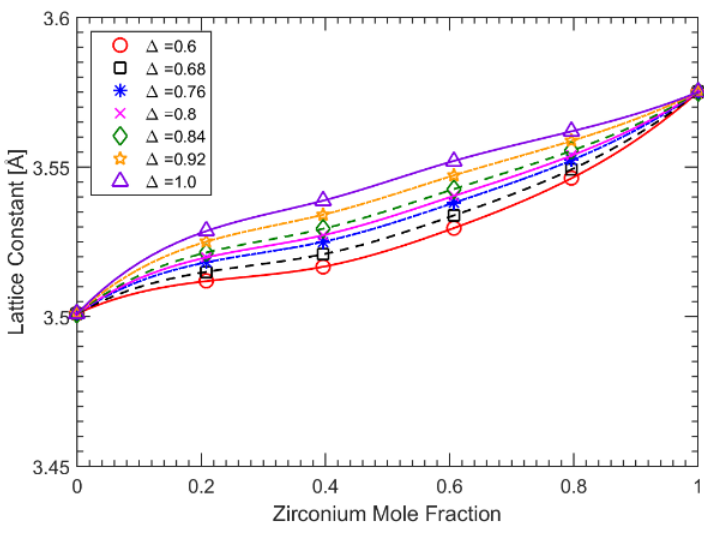

(b)

Figure 12: Sensitivity analysis of U-Zr alloy MEAM parameter $\Delta$ on (a) enthalpy of mixing and (b) Vegard's law.

Figure 12 shows how the alloy parameter $\Delta$ affects the alloy's thermal properties. It can be seen that the change in the parameter affects the change in both the enthalpy of mixing and the Vegard's law mostly via a symmetric parabola, and only slightly changes the shape of the curve, indicating a $+\mathrm{P}$ and $-\mathrm{S}$ relationship. This parameter shows great potential for calculated MEAM alloy property adjustment using only a symmetric parabola. 


\section{Fitting the MEAM Potential}

While either the force matching method (34-37) or particle swarm optimization (PSO) (38-41) has been commonly used to fit EAM and MEAM potentials, both can have difficulties when used with high temperature transition metals. Therefore, the potentials were iterated by using knowledge of the system.

To construct a high temperature MEAM potential, it is still necessary to begin with the $0 \mathrm{~K}$ density functional theory (DFT) or experimental results in order to ensure that the basis of the potential matches the known elastic constants and defect formation energies. The procedure for fitting a 1NN MEAM potential is described by Baskes et al. $(17,42)$. After a 1NN MEAM has been made, the transition to a $2 \mathrm{NN}$ MEAM involves iterating the screening parameter $\mathrm{C}_{\min }$, $A$, and the partial electron density parameters $t^{1}, t^{2}, t^{3}, \beta^{0}, \beta^{1}, \beta^{2}$ and $\beta^{3}$.

It is useful to fit properties based on symmetry of the defects, elastic constants, or interface, as well as the symmetry of the phase. In addition, as noted previously, different crystal structure properties are dependent on different parameters, making the fitting of multiple phases useful as well. The electron orbital in spherical harmonics can help visualize some of the symmetries of the orthogonal Legendre polynomials. This knowledge combined with the sensitivity tables in the proceeding sections enables the fitting of new elemental and alloy systems employing knowledge of the sensitivity of the desired property to the variation of each parameter.

A flow diagram of the fitting methodology can be found in Figure 13. The flow outlines a simple iterative methodology for fitting various potentials. The process begins with creating databases of properties to be fit to the MEAM potential, and then moves onto the order in which the MEAM potential is checked on how it matches the values in the created database. It is important to start with the simplest ground state properties first, ensuring the base of the potential has a good starting point, after which the thermal properties are fit via the iterative process seen in Figure 13. This process is done first for the elemental MEAM parameters and then for the alloy parameters. However, as shown in the sensitivity analysis above, both the elemental and alloy parameters may have to be adjusted in order to fit the alloy properties of interest.

The created elemental and alloy databases of properties to match the MEAM potential to should include properties with significantly different local symmetries (e.g. vacancies and stacking fault energies) in the hopes of creating a versatile and transferable potential. The elemental database often includes properties like elastic constants, bulk modulus, cohesive energy, migration energies, surface energies, stacking fault energies, crystal phase energies, lattice parameter, thermal expansion, Grüneisen parameter, heat capacity, phase transition temperatures and pressures, melting temperatures and more. The alloy database often includes elastic constants, bulk modulus, enthalpy of formation, lattice constants, and known thermal properties for the chosen reference phase, any ordered alloys, and any intermetallics in the system. 


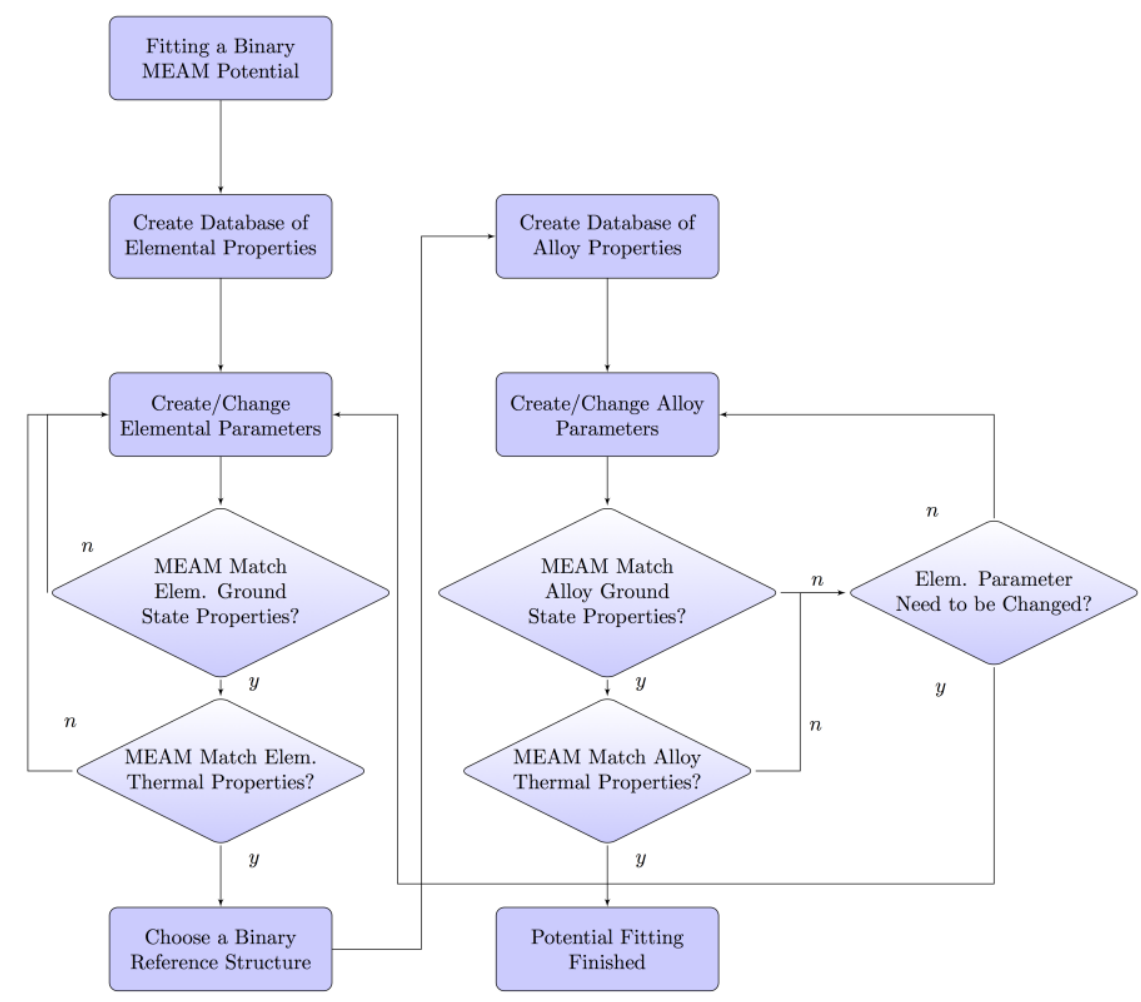

Figure 13: MEAM potential fitting flow diagram, showing the process of fitting a MEAM binary alloy system.

\section{Conclusions}

A sensitivity analysis was conducted using OAT sampling of the MEAM interatomic potential parameters and how changes in parameters propagate throughout the system, causing changes to numerous structural and thermodynamic properties. This analysis is key to unravelling the cause/effect relationship of interatomic potentials, as well as discovering their benefits and drawbacks. This sensitivity analysis shows how physical properties can be fitted when using interatomic potentials, as well as the properties which cannot be adjusted. This research gives insight into the overall transferability and uncertainty when fitting and using interatomic potentials such as the MEAM.

The analysis begins on the 13 elemental MEAM parameters and how they affect the bcc ground state elastic constants and relative phase energies. The sensitivity analysis performed is shown to agree well with previously published results. However, our analysis takes the sensitivity of the ground state properties a bit further by including more parameters that were varied, adding a quantitative indication of the significance of a change, and adding the directionality of the parameter effects on the observed properties.

Next, the effect the elemental parameters had on the thermal properties is examined. The trends in which MEAM parameters affect the thermal properties of the bcc metals have been successfully analyzed and categorized. The parameter effects are often broken up into those with more or less direct dependence on their local environment. The thermal properties examined include heat capacity, melting point temperature, thermal expansion, and lattice constant. Once again a quantitative number was introduced to gauge the significance of the change as well as the directionality, giving understanding of the role parameters have on atomistic properties. The 
linearity of the change was examined as well. This is useful because it can help facilitate constructing new MEAM potentials and help to understand how parameters cause changes to the properties. The melting point, lattice constant, and thermal expansion all seem to be moderately dependent on a number of MEAM parameters. However, the heat capacity was shown to have little variance with many of the properties and could not be easily adjusted. The parameters that had much of any significance on the values $E_{C}$ and $A$ are usually fit to ground state properties of cohesive energy and relative phase stability.

Then the effect the elemental parameters have on alloys was examined. The alloy properties examined were the Vegard's law for lattice constants and the enthalpy of mixing. The sensitivity analysis of alloy properties has never been previously published, and therefore a method of deconstructing the effects was developed. The method developed allowed the change to be broken up by magnitude the strength or length of bonding between the atoms changed and how the elements were being bonded together changed. Again the directionality of the change in the alloy property curves was also documented. From this analysis it was determined that before alloy parameters are fit, it is important that the elemental parameters be fit well first, as many of the alloy properties can change significantly with changes to the elemental parameters.

The effect the alloy parameters had on the alloy properties was examined. The alloy properties examined include Vegard's law for lattice constants and the enthalpy of mixing. The analysis showed that a few key parameters exist that allow for shifts in either the Vegard's law curve or enthalpy of mixing curve, like the alloy $\Delta$ and $\alpha$ parameters, making the fitting of most alloy thermodynamics relatively simple.

Finally, the fitting of MEAM potentials was briefly discussed. A flow chart is given to help facilitate the development of new interatomic potentials. The previous tables and data can help future development of interatomic potentials by understanding how changes in parameters affect a multitude of properties, where many of the previous fitting procedures relied on a guess and check method that has the potential to break down outside the range that was directly fitted. This research will allow the further development of these potentials, and gives insight allowing improved fitting procedures.

\section{Acknowledgments}

Authors acknowledge support from an Idaho National Laboratory (INL), a National Science Foundation - Domestic Nuclear Detection Office (NSF-DNDO) Academic Research grant, and from the Department of Energy Nuclear Energy University Program (DOE-NEUP).

\section{Appendix A: 2NN MEAM Theory}

The MEAM potential builds on its predecessor (the EAM potential) by allowing the background electron density to depend on the local environment via angularly dependent partial electron densities.

The MEAM potential for a single element contains 13 adjustable parameters $\left(\mathrm{A}, \mathrm{B}^{0}, \mathrm{~B}^{1}\right.$, $\mathrm{B}^{2}, \mathrm{~B}^{3}, \mathrm{t}^{1}, \mathrm{t}^{2}, \mathrm{t}^{3}, \alpha, \delta, \mathrm{E}_{\mathrm{C}}, \mathrm{C}_{\min }$, and $\left.\mathrm{C}_{\max }\right)$ used to obtain the physical properties seen by experiments or ab-initio simulations. The MEAM potential total energy $U$ of a system of atoms is given by

$$
\mathrm{U}=\sum_{i} F_{i}\left(\overline{\rho_{l}}\right)+\frac{1}{2} \sum_{i, j \neq i} \phi_{i j}\left(R_{i j}\right) S\left(R_{i j}\right)
$$


where $F_{i}$ is the embedding function, $\rho_{i}$ is the background electron density at site $\mathrm{i}, \mathrm{S}_{\mathrm{ij}}$ is the radial screening function, and $\varphi_{\mathrm{ij}}\left(\mathrm{R}_{\mathrm{ij}}\right)$ is the pair interaction function between atoms $\mathrm{i}$ and $\mathrm{j}$ at a distance, $R_{\mathrm{ij}}$.

The embedding function describes the energy it takes to add (or embed) the atom to the crystal structure. The embedding function for the MEAM potential is defined by

$F_{i}\left(\bar{\rho}_{i}\right)=A_{i} E_{c i}\left(\frac{\bar{\rho}_{i}}{\bar{\rho}_{l}^{e}}\right) \ln \left(\frac{\bar{\rho}_{i}}{\bar{\rho}_{l}^{e}}\right)$,

where $\mathrm{A}_{\mathrm{i}}$ is an adjustable parameter, $\mathrm{E}_{\mathrm{Ci}}$ is the cohesive energy, and $\rho^{e}{ }_{i}$ is the electron density evaluated at equilibrium in the reference structure.

The electron density $(\rho)$ is dependent upon the local environment, in particular the angular relation between atoms. These angular contributions are split into partial electron densities. The geometric manner in which $\rho_{i}^{(l)}$ is defined allows us to consider the partial background electron densities in a similar manner to the specific angular momentum contributions to the background electron densities known as electron orbitals (spdf). The background electron density $(\bar{\rho})$ is composed of a spherically symmetrical partial electron density $\bar{\rho}_{i}^{(0)}$ and angular partial electron densities $\bar{\rho}_{i}^{(1)}, \bar{\rho}_{i}^{(2)}$ and $\bar{\rho}_{i}^{(3)}$, that are comprised of orthogonal Legendre polynomials.

$$
\left(\rho_{i}^{(l)}\right)^{2}=\sum_{j \neq i} \sum_{k \neq i} \rho_{j}^{a(l)}\left(R_{i j}\right) \rho_{k}^{a(l)}\left(R_{i k}\right) P_{l}^{0}\left(\cos \left(\theta_{i k j}\right)\right)
$$

In these equations, $\rho_{j}^{a(h)}$ is the atomic electron densities of atom $\mathrm{j}$ at the distance $\mathrm{R}_{\mathrm{ij}}$ relative to the site $i$. The atomic electron densities are given by

$\rho_{j}^{a(h)}\left(R_{i j}\right)=\rho_{0} S_{i j} f_{c u t}\left(R_{i j}\right) \exp \left[-\beta^{(h)}\left(\frac{R_{i j}}{r_{e}}-1\right)\right]$,

where $\beta^{(h)}$ is the partial electron density decay, $r_{e}$ is the nearest neighbor distance, $S_{\mathrm{ij}}$ is the screening factor, $\mathrm{f}_{\text {cut }}$ is the cut-off function, and $\rho_{0}$ is a scaling factor which plays no role for pure elements, but has a significant role for alloy systems. The total background electron density can be obtained from the weighted partial background electron densities, i.e.,

$$
\bar{\rho}_{i}=\rho_{i}^{(0)} \sqrt{1+\sum_{l=0}^{3} t_{i}^{(l)}\left(\frac{\rho_{i}^{(l)}}{\rho_{i}^{(0)}}\right)^{2}}
$$

(AErr

\section{or! Bookmark not defined.)}

where $t_{i}^{(l)}$ is the weighting factor for each of the partial electron densities. For convenience, $t^{(0)}=1$. Setting one value helps to visualize changes in the weighted parameters since the electron density is weighted by the $t_{i}$ parameters. 
Screening between two atoms ( $\mathrm{i}$ and $\mathrm{j}$ ) is defined as the product of screening factors, $\mathrm{S}_{\mathrm{ijk}}$, due to the neighboring atoms $(\mathrm{k})$ that contribute to screening, leading to

$S_{i j}=\prod_{k \neq i, j} S_{i j k}$

The three-dimensional screening is captured using an ellipsoidal shape function, where atoms $\mathrm{i}$ and $\mathrm{j}$ are being screened by atom $\mathrm{k}$. The amount of screening is separated into three regions: completely screened, partially screened, and non-screened.

The ellipse parameter $\mathrm{C}$ is determined by a function of ratios between atoms $\mathrm{i}, \mathrm{j}$, and $\mathrm{k}$, given by

$C=\frac{2\left(X_{i k}+X_{k j}\right)-\left(X_{i k}-X_{k j}\right)^{2}-1}{1-\left(X_{i k}-X_{k j}\right)^{2}}$

where $X_{i k}=\left(R_{i k} / R_{i j}\right)^{2}$ and $X_{k j}=\left(R_{k j} / R_{i j}\right)^{2}$. The screening factor $\mathrm{S}_{\mathrm{ikj}}$ is defined as

$S_{i k j}=f_{c}\left[\left(C-C_{\min }\right) /\left(C_{\max }-C_{\min }\right)\right]$,

where $C_{\min }$ and $C_{\max }$ determine the extent of the screening and $f_{C}$ is the cut-off function.

The cutoff function describes the smooth, gradually decreasing distance effect on the interactions between the atoms. The smooth cut-off screening function (fc) is defined by

$f_{c}(x)=1, \quad x \geq 1$

$\left[1-(1-x)^{6}\right]^{2}, \quad 0<x<1$,

$0, \quad x \leq 0$

$x=\left(\frac{R_{i j}^{c u t-o f f}-R_{i j}}{\Delta R_{i j}}\right)$

where $\Delta \mathrm{R}_{\mathrm{ij}}$ is the width of the cut-off region and $\mathrm{R}^{\text {cut-off }}$ is the maximum cut-off distance. Normally, the MEAM cut-off function employs $(1-\mathrm{x})^{4}$ rather than the $(1-\mathrm{x})^{6}$; however, the power was changed to the sixth when fitting the stacking fault energy for $\mathrm{Zr}$.

The pair potential, instead of being given explicitly, is given as a functional of an embedding atom function and a universal function $\left(\mathrm{E}^{\mathrm{u}}(\mathrm{R})\right)$ that is able to reproduce the universal equation of state (EOS) (43) for the potential energy of a reference crystal structure. The universal function $\left(E^{\mathrm{u}}(\mathrm{R})\right)$ describes the uniform expansion or contraction in the reference structure. However, the 2NN MEAM adds additional complexity, since both the 1NN and 2NN atoms need to be considered in the reference structure. The 1NN MEAM is able to neglect the $2 \mathrm{NN}$ interactions through the use of a strong many-body screening function. However, the $2 \mathrm{NN}$ MEAM is able to include $2 \mathrm{NN}$ interactions by adjusting the many-body screening function so that it becomes less severe, i.e.,

$E^{u}\left(R_{i j}\right)=F\left[\overline{\rho^{0}}\left(R_{i j}\right)\right]+\frac{1}{2} \sum_{i, j} \phi\left(R_{i j}\right) S\left(R_{i j}\right)$ 
where $\mathrm{E}^{\mathrm{u}}\left(\mathrm{R}_{\mathrm{ij}}\right)$ is the EOS. The EOS can be expanded using the relation obtained by Rose et al. (43) as a function of nearest-neighbor distance $\mathrm{R}$, and the pair potential and screening function can be broken up into the $1 \mathrm{NN}$ and $2 \mathrm{NN}$ components, i.e.,

$$
\begin{aligned}
& -E_{c}\left[1+a^{*}+\delta\left(a^{*}\right)^{3} \frac{r_{e}}{R_{i j}}\right] e^{-a^{*}}=F\left[\overline{\rho^{0}}\left(R_{i j}\right)\right]+\left(\frac{Z_{1}}{2}\right) \phi\left(R_{i j}\right)+\left(\frac{Z_{2} S}{2}\right) \phi\left(a R_{i j}\right) \\
& a^{*}=\alpha\left(R_{i j} / r_{e}-1\right) \\
& \alpha=\left(9 B \Omega / E_{c}\right)^{1 / 2}
\end{aligned}
$$

here, $Z_{2}$ is the number of $2 n d N N$ atoms, "a" is the ratio between the second and first nearestneighbor distances, and $\mathrm{S}$ is the screening function on the $2 \mathrm{NN}$ interactions. It should be noted that the screening function $\mathrm{S}$ is a constant for a given reference structure, if a value is given to $\mathrm{C}_{\max }$ and $\mathrm{C}_{\min }$. Equation (A11) cannot be directly solved. Therefore, another pair potential, $\psi(\mathrm{R})$, was introduced that allows for an iterative solution. Equation (A11) can now be written as

$E^{u}\left(R_{i j}\right)=F\left[\overline{\rho^{0}}\left(R_{i j}\right)\right]+\left(\frac{Z_{1}}{2}\right) \psi\left(R_{i j}\right)$

$\psi\left(R_{i j}\right)=\phi\left(R_{i j}\right)+\left(\frac{z_{2} S}{Z_{1}}\right) \phi\left(a R_{i j}\right)$.

The function $\psi(\mathrm{R})$ can be calculated from the equation as a function of $\mathrm{R}_{\mathrm{ij}}$. Then, the pair potential $\phi\left(R_{\mathrm{ij}}\right)$ is calculated using

$\phi\left(R_{i j}\right)=\psi\left(R_{i j}\right)+\sum_{n=1}(-1)^{n}\left(\frac{Z_{2} S}{Z_{1}}\right)^{n} \psi\left(a^{n} R_{i j}\right)$.

The summation in Equation (A13) is performed until the correct value of energy is obtained for the equilibrium reference structure (44).

\section{$\underline{2^{\text {nd }} \text { Nearest Neighbor (2NN) MEAM Alloy }}$}

The MEAM potential becomes more complex for binary and tertiary alloys. A binary alloy has 14 adjustable parameters for each element and at least 15 adjustable parameters $(\delta, \alpha$, $\left.\rho_{\mathrm{U}}, \rho_{\mathrm{Zr}}, \Delta, \mathrm{C}_{\min }{ }^{(1,1,2)}, \mathrm{C}_{\min }{ }^{(1,2,1)}, \mathrm{C}_{\min }{ }^{(1,2,2)}, \mathrm{C}_{\min }{ }^{(2,1,2)}, \mathrm{C}_{\max }{ }^{(1,1,2)}, \mathrm{C}_{\max }{ }^{(1,2,1)}, \mathrm{C}_{\max }{ }^{(1,2,2)}, \mathrm{C}_{\max }{ }^{(2,1,2)}\right)$ for the binary alloy interactions. It should be noted that the $\rho$ can be considered an alloy parameter because it does not change the elemental properties but only the alloy properties.

To describe an alloy system, the pair interaction between different elements should be determined. In the 2NN MEAM, a perfectly ordered binary intermetallic compound, where only one type of atom has different atoms as $1 \mathrm{NN}$, is considered to be a good reference structure for creating the alloy pair potential. The $\mathrm{B} 1(\mathrm{NaCl}$ type) reference structure is used for the $\mathrm{U}-\mathrm{Zr}$ MEAM potential. For the B1 reference structure, the total energy per atoms (for half $\mathrm{i}$ atoms and half $\mathrm{j}$ atoms), $E_{i j}^{u}(R)$, is given by

$E_{i j}^{u}(R)=\frac{1}{2}\left\{F_{i}\left(\bar{\rho}_{i}\right)+F_{j}\left(\bar{\rho}_{j}\right)+Z^{i j} \phi_{i j}(R)+\frac{1}{2} Z_{2}^{i j}\left(\phi_{i i}(a R)+\phi_{j j}(a R)\right)\right\}$, 
where $Z^{\mathrm{ij}}$ is the number of $2 \mathrm{NN}$ atoms in the reference structure, $\phi_{\mathrm{ii}}$ and $\phi_{\mathrm{jj}}$ are pair interactions between $\mathrm{i}$ atoms and between $\mathrm{j}$ atoms, respectively, and $\mathrm{a}$ is the ratio between the second and first nearest-neighbor distances. The procedure for computing $\rho_{\mathrm{i}}$ and $\rho_{\mathrm{j}}$ is not different from that in 1NN MEAM except that the contribution from the second nearest-neighbors should also be considered. The pair interactions between the same types of atoms can be computed from the descriptions of individual elements (44), i.e.,

$\phi_{i j}(R)=\frac{1}{Z_{i j}}\left\{2 E_{i j}^{u}(R)-F_{i}\left(\bar{\rho}_{i}\right)-F_{j}\left(\bar{\rho}_{j}\right)-\frac{1}{2} Z_{2}^{i j}\left(\phi_{i i}(a R)+\phi_{j j}(a R)\right)\right\}$.

The cohesive energy for the alloy is determined by the elemental cohesive energies and a formation parameter $\Delta_{\mathrm{ij}}$, i.e.,

$E_{i j}^{0}=\frac{\left(E_{i}^{0}+E_{j}^{0}\right)}{2}-\Delta_{i j}$

\section{References}

1. M. P. Allen, D. J. Tildesley, Computer simulation of liquids. (Oxford university press, 1989).

2. D. Brenner, The art and science of an analytic potential. physica status solidi(b) 217, 2340 (2000).

3. D. Frenkel, B. Smit, Understanding molecular simulation: from algorithms to applications. Computational sciences series 1, 1-638 (2002).

4. R. LeSar, Introduction to computational materials science: fundamentals to applications. (Cambridge University Press, 2013).

5. S. B. Sinnott, D. W. Brenner, Three decades of many-body potentials in materials research. Mrs Bulletin 37, 469-473 (2012).

6. P. Brommer, F. Gähler, Potfit: effective potentials from ab initio data. Modelling and Simulation in Materials Science and Engineering 15, 295 (2007).

7. S.-G. Kim et al., Semi-Empirical Potential Methods for Atomistic Simulations of Metals and Their Construction Procedures. Journal of Engineering Materials and Technology 131, 041210 (2009).

8. M. Malshe et al., Parametrization of analytic interatomic potential functions using neural networks. The Journal of chemical physics 129, 044111 (2008).

9. A. P. Thompson, L. P. Swiler, C. R. Trott, S. M. Foiles, G. J. Tucker, Spectral neighbor analysis method for automated generation of quantum-accurate interatomic potentials. Journal of Computational Physics 285, 316-330 (2015).

10. M. A. Tschopp et al., Generalized framework for interatomic potential design: Application to Fe-He system. Journal of Nuclear Materials 425, 22-32 (2012).

11. G. Iaccarino, "Quantification of uncertainty in flow simulations using probabilistic methods," (DTIC Document, 2009).

12. M. T. Reagan, H. Najm, P. Pebay, O. Knio, R. Ghanem, Quantifying uncertainty in chemical systems modeling. International journal of chemical kinetics 37, 368-382 (2005).

13. A. Saltelli et al., Global sensitivity analysis: the primer. (John Wiley \& Sons, 2008). 
14. M. Finnis, J. Sinclair, A simple empirical N-body potential for transition metals. Philosophical Magazine A 50, 45-55 (1984).

15. A. P. Moore, B. Beeler, C. Deo, M. I. Baskes, M. A. Okuniewski, Atomistic modeling of high temperature uranium-zirconium alloy structure and thermodynamics. Journal of Nuclear Materials, (2015).

16. A. P. Moore, C. Deo, M. I. Baskes, M. A. Okuniewski, Atomistic mechanisms of morphological evolution and segregation in U-Zr alloys. Acta Materialia 115, 178-188 (2016).

17. M. Baskes, Modified embedded-atom potentials for cubic materials and impurities. Physical Review B 46, 2727 (1992).

18. M. S. Daw, M. I. Baskes, Embedded-atom method: Derivation and application to impurities, surfaces, and other defects in metals. Physical Review B 29, 6443 (1984).

19. B. Blumenthal, The transformation temperatures of high-purity uranium. Journal of Nuclear Materials 2, 23-30 (1960).

20. Y.-M. Kim, N. J. Kim, B.-J. Lee, Atomistic Modeling of pure Mg and Mg-Al systems. Calphad 33, 650-657 (2009).

21. B.-J. Lee, A modified embedded atom method interatomic potential for silicon. Calphad 31, 95-104 (2007).

22. B.-J. Lee, J.-H. Shim, M. Baskes, Semiempirical atomic potentials for the fcc metals $\mathrm{Cu}$, $\mathrm{Ag}, \mathrm{Au}, \mathrm{Ni}, \mathrm{Pd}, \mathrm{Pt}, \mathrm{Al}$, and $\mathrm{Pb}$ based on first and second nearest-neighbor modified embedded atom method. Physical Review B 68, 144112 (2003).

23. M. Horstemeyer et al., Hierarchical bridging between ab initio and atomistic level computations: Calibrating the modified embedded atom method (meam) potential (part a). JOM 67, 143-147 (2015).

24. J. Hughes et al., Hierarchical bridging between ab initio and atomistic level computations: Sensitivity and uncertainty analysis for the modified embedded-atom method (meam) potential (part b). JOM 67, 148-153 (2015).

25. S. Foiles, M. S. Daw, Computer code DYNAMO. (Sandia National Labratories, unpublished).

26. B.-J. Lee, M. Baskes, Second nearest-neighbor modified embedded-atom-method potential. Physical Review B 62, 8564 (2000).

27. B. Beeler, C. Deo, M. Baskes, M. Okuniewski, Atomistic properties of $\gamma$ uranium. Journal of Physics: Condensed Matter 24, (2012).

28. P. Söderlind, First-principles elastic and structural properties of uranium metal. Physical Review B 66, 085113 (2002).

29. H. Wangyu et al., Calculation of thermodynamic properties of $\mathrm{Mg}-\mathrm{RE}(\mathrm{RE}=\mathrm{Sc}, \mathrm{Y}, \mathrm{Pr}$, $\mathrm{Nd}, \mathrm{Gd}, \mathrm{Tb}, \mathrm{Dy}, \mathrm{Ho}$ or Er) alloys by an analytic modified embedded atom method. Journal of Physics D: Applied Physics 33, 711 (2000).

30. J. Kim, Y. Koo, B.-J. Lee, Modified embedded-atom method interatomic potential for the Fe-Pt alloy system. Journal of Materials Research 21, 199-208 (2006).

31. Y.-M. Kim, B.-J. Lee, A semi-empirical interatomic potential for the $\mathrm{Cu}-\mathrm{Ti}$ binary system. Materials Science and Engineering: A 449-451, 733-736 (2007).

32. I. Sa, B.-J. Lee, Modified embedded-atom method interatomic potentials for the $\mathrm{Fe}-\mathrm{Nb}$ and Fe-Ti binary systems. Scripta Materialia 59, 595-598 (2008). 
33. J. Luyten, J. De Keyzer, P. Wollants, C. Creemers, Construction of modified embedded atom method potentials for the study of the bulk phase behaviour in binary Pt-Rh, Pt-Pd, Pd-Rh and ternary Pt-Pd-Rh alloys. Calphad 33, 370-376 (2009).

34. Y. Li, D. J. Siegel, J. B. Adams, X.-Y. Liu, Embedded-atom-method tantalum potential developed by the force-matching method. Physical Review B 67, 125101 (2003).

35. S. Ryu, C. R. Weinberger, M. I. Baskes, W. Cai, Improved modified embedded-atom method potentials for gold and silicon. Modelling and Simulation in Materials Science and Engineering 17, 075008 (2009).

36. D. Smirnova, S. Starikov, V. Stegailov, Interatomic potential for uranium in a wide range of pressures and temperatures. Journal of Physics: Condensed Matter 24, 015702 (2012).

37. D. Smirnova et al., A ternary EAM interatomic potential for U-Mo alloys with xenon. Modelling and Simulation in Materials Science and Engineering 21, 035011 (2013).

38. Z. Cui, F. Gao, Z. Cui, J. Qu, A second nearest-neighbor embedded atom method interatomic potential for Li-Si alloys. Journal of Power Sources 207, 150-159 (2012).

39. Z. Cui, F. Gao, Z. Cui, J. Qu, Developing a second nearest-neighbor modified embedded atom method interatomic potential for lithium. Modelling and simulation in materials science and engineering 20, 015014 (2012).

40. M. I. Pascuet, G. Bonny, J. R. Fernández, Many-body interatomic U and Al-U potentials. Journal of Nuclear Materials 424, 158-163 (2012).

41. Y. Li, T.-R. Shan, T. Liang, S. B. Sinnott, S. R. Phillpot, Classical interatomic potential for orthorhombic uranium. Journal of Physics: Condensed Matter 24, 235403 (2012).

42. M. Baskes, R. Johnson, Modified embedded atom potentials for HCP metals. Modelling and Simulation in Materials Science and Engineering 2, 147 (1994).

43. J. H. Rose, J. R. Smith, F. Guinea, J. Ferrante, Universal features of the equation of state of metals. Physical Review B 29, 2963 (1984).

44. B.-J. Lee, M. Baskes, H. Kim, Y. K. Cho, Second nearest-neighbor modified embedded atom method potentials for bcc transition metals. Physical Review B 64, 184102 (2001). 